\title{
Ethics in psychosocial and biomedical research - A training expe- rience at the Interdisciplinary Center for Bioethics (CIEB) of the University of Chile
}

\author{
Fernando Lolas ${ }^{2}$, Eduardo Rodriguez ${ }^{3}$
}

\begin{abstract}
This paper reviews the experience in training Latin American professionals and scientists in the ethics of biomedical and psychosocial research at the Interdisciplinary Center for Studies in Bioethics (CIEB) of the University of Chile, aided by a grant from Fogarty International Center (FIC) - National Institutes of Health from 2002 to 2011. In these 10 years of experience, 50 trainees have completed a 12-month training combining on-line and in-person teaching and learning activities, with further support for maintaining contact via webmail and personal meetings. The network formed by faculty and former trainees has published extensively on issues relevant in the continent and has been instrumental in promoting new master level courses at different universities, drafting regulations and norms, and promoting the use of bioethical discourse in health care and research. Evaluation meetings have shown that while most trainees did benefit from the experience and contributed highly to developments at their home institutions and countries, some degree of structuring of demand for qualified personnel is needed in order to better utilize the human resources created by the program. Publications and other deliverables of trainees and faculty are presented.
\end{abstract}

Keywords: Bioethics, Ethics training, Biomedical research, Psychosocial research

\section{Ética en investigación biomédica y psicosocial - Experiencia de capacitación en el Centro Inter- disciplinario de Estudios en Bioética (CIEB) de la Universidad de Chile}

Resumen: El presente artículo revisa la experiencia de capacitación en ética de investigación biomédica y psicosocial a profesionales y científicos latinoamericanos en el Centro Interdisciplinario de Estudios en Bioética de la Universidad de Chile, con el apoyo de un grant del Centro Internacional Fogarty de los Institutos Nacionales de la Salud de Estados Unidos, de 2002 a 2011. En estos 10 ańos de experiencia, 50 profesionales han completado una capacitación de 12 meses, que combina actividades de aprendizaje en línea y presenciales con apoyo vía email y tutoría. La red, formada por los académicos del programa y los participantes, ha publicado extensamente en temas relevantes para el continente y ha sido instrumental en promover cursos de nivel de magíster en diferentes universidades, proponer regulaciones y promover el uso del discurso bioético en la atención de salud y la investigación. Los encuentros de evaluación han demostrado que, aun cuando la mayoría de los participantes se beneficiaron de la experiencia y contribuyeron grandemente al desarrollo en sus instituciones y su país, se necesita algún grado de estructuración para la demanda de personal calificado que pueda utilizar mejor los recursos humanos creados por el programa. Se presentan las publicaciones y otros productos de académicos y participantes en el programa.

Palabras clave: bioética, capacitación en ética, investigación biomédica, investigación psicosocial

\section{Ética na pesquisa biomédica e psicossocial - Experiência de capacitação no Centro Interdisci- plinar de Estudos em Bioética (CIEB) da Universidade do Chile}

\begin{abstract}
Resumo: O presente artigo revisa a experiência de capacitação em ética da pesquisa biomédica e psicossocial a profissionais e cientistas latino-americanos no Centro Interdisciplinar de Estudos em Bioética da Universidade do Chile, com o apoio de bolsa do Centro Internacional Fogarty dos Institutos Nacionais de Saúde dos Estados Unidos, de 2002 a 2011 . Nestes 10 anos de experiência, 50 profissionais completaram uma capacitação de 12 meses, que combina atividades de aprendizagem "on line" e presenciais com apoio via e-mail e tutoria. A rede formada por acadêmicos do programa e participantes publicou extensamente temas relevantes para o continente e foi instrumental em promover cursos em nivel de mestrado em diferentes universidades, propor regulamentaçóes e promover o uso do discurso bioético na atenção à saúde e à pesquisa. Os encontros de avaliação têm demonstrado que, mesmo que a maioria dos participantes tenha se beneficiado da experiência e contribuido grandemente para o desenvolvimento de suas instituiçóes e seu país, ainda necessita de algum grau de estruturação para a demanda de pessoal qualificado que possa utilizar melhor os recursos humanos criados pelo programa. Sáo apresentados publicaçóes e outros produtos de acadêmicos e participantes do programa.
\end{abstract}

Palavras-chave: bioética, capacitação em ética, pesquisa biomédica, pesquisa psicossocial

\footnotetext{
${ }^{1}$ During the preparation of this article, the first author received support from Grant NIH-Fogarty R25 TW6056 and the Humboldt Foundation, Germany.

${ }^{2}$ Director, Interdisciplinary Center for Studies in Bioethics. Professor, Dept. of Psychiatry, Faculty of Medicine, University of Chile, Chile. Principal Investigator, Grant NIH-Fogarty R25 TW6056.

Correspondence: flolas@u.uchile.cl
}

${ }^{3}$ Coordinator, Training Program on Research Ethics, Chile 


\section{Background}

The University of Chile, the oldest public university in Chile and the most accredited research institution in the country, ranking among the most productive and solvent in the Region of the Americas and the Caribbean, has pioneered in the establishment of new fields of inquiry. Bioethics was among them.

The Interdisciplinary Center for Studies on Bioethics (CIEB) was established in 1993(1) and in 1994 it entered into collaboration with the Regional Program on Bioethics established by an agreement between the Chilean Government, the University of Chile and the Pan American Health Organization (PAHO). The latter, with several burocratic vicissitudes, lasted until March 2010.

Throughout its existence, CIEB has been instrumental in promoting the dissemination of bioethical discourses and practices in the website www. bioetica.uchile.cl and through the indexed, peerreviewed journal Acta Bioethica (ISSN 07175906), published with support from PAHO until 2010 and from 2011 onwards in electronic format (www.actabioethica.cl). CIEB also edits books, congress proceedings and monographic issues of Acta Bioethica, freely available at the websites.

CIEB also participates in seminars, congresses, and workshops in Latin America, the USA, and Europe and conducts research projects supported by private and governmental sources, such as the Ford Foundation, the Rockefeller Foundation, the US Department of Energy, the Chilean Research Commission, the Alexander von Humboldt Foundation and the US National Institutes of Health (NIH).

This paper reviews one long lasting project supported by the National Institutes of Health (NIH) through grants from the Fogarty International Center in collaboration with other Institutes. For 10 years, from 2002 thru 2011, the emphasis has been placed on training selected professionals and academics from Latin America and the Caribbean in biomedical and psychosocial research ethics.
Topics covered have been diverse and recruitment of trainees encouraged multidisciplinarity and differences in outlooks. Throughout the years, with different emphases, the main goal has been to help increase the number of persons familiar with the ethics of research in the biomedical and psychosocial disciplines and to foster the development of training programs at different institutions of the continent. This is important since previous projects have depended on help external to the cultural milieu of the Latin American countries and the need to establish a "culture-fair" bioethics was felt by individuals and institutions.

\section{Curricular development in research ethics - a culture-sensitive approach}

Despite improvements in research output in the biological, social, and medical sciences, several problems remain, with ethical underpinnings that justify training in research bioethics in the Region of South and Central America. Some aspects relevant to a training program in research ethics were identified and provided the rationale for this training program.

\section{The 10/90 gap}

Enhancements in global health status have been accompanied by a widening health and poverty gap between countries. Most of the funds allotted to research continue to focus on the health problems of the world's richest populations. Attending to this disparity, known as the 10/90 gap, the Global Forum for Health Research recommended that resource-poor countries should strengthen management of health research so as to meet their national needs as well as contribute to global knowledge. Increase in research output naturally demands sound ethical oversight.

\section{Globalization and resource-poor countries}

The trend to globalization in clinical and psychosocial research implies that sponsors in economically advanced countries outsource trials and research to poor communities within developed countries or, most frequently, to resource- 
poor nations. The cost of conducting research is less than in advanced countries. Poor countries offer large patient pools for diseases, thus ensuring rapid recruitment and reduction of time for completing trials. Regulations are weak or nonexistent, and lack of qualified policymakers and scientists create an imbalance between what is offered to communities and the actual benefits in terms of manpower development or economic support. Public awareness about the need to ethical control of research is imperative.

\section{The know-do gap}

Another aspect that merits consideration is the so called "know-do gap". While information is available on many health-related issues, its conversion to policy and practical measures is delayed precisely in those countries where its application would make a difference in living conditions. This is not only a matter of economic importance. Its ethical dimension arises from the fact that resource-poor countries- or some privileged groups within them-consider that they have the right to enjoy the highest possible standard derived from scientific progress. Only a cadre of professionals conversant with the state of the art in research and also aware of the value-laden nature of scientific knowledge can enter into a dialogue with experts and lay people regarding the appropriateness and timeliness of investments in the conversion of data into practice. On the other hand, an equitable distribution of the benefits derived from scientific research is a matter for ethical reflection by the scientific community, policymakers, and public at large.

\section{Research: cultural implications and responsible conduct}

Research is a context-dependent, patterned cultural process. Some activities called research in less developed countries would not qualify as such in scientifically alphabetized countries. Social demand for research (as different from need or desire) continues to be low in Latin America, as reflected in resource allocation, social recognition, and employment opportunities.
Ethical considerations affect quality of research and opportunities for collaborative work. In many Latin American countries informed consent, responsible conduct of research, risk assessment, protection from harm, privacy and confidentiality, equitable opportunities for subjects, and avoidance of stigmatization (among other issues) are still more a matter of compliance with donor agencies than a felt need of the scientific community. A combination of face-to-face and distant training within the same cultural community has proven appropriate for dealing with these issues. Ethics is always local, despite the fact that research may be considered a universal intellectual enterprise.

\section{Social roles: clinician and/or researcher}

Scarcity of personnel, professional traditions, and restricted funding are responsible for confusion between the roles of caregiver and researcher in many Latin American settings. Pervasive "therapeutic misunderstandings" $(1,2)$, inadequate notions of "standard of care", different contextual interpretation of guidelines stemming from other cultures, and discrepancies between researchers from different countries/traditions/professions militate against a unified view of the research enterprise that could be applied to improve quality and train scholars. This training program confronted trainees with real-life examples of research in specific contexts and/or in response to felt needs of populations and institutions. Special emphasis given to public health topics and anthropological analysis was dictated by the priorities of healthcare systems in the continent and the multicultural, pluralistic environments in which researchers must act.

\section{International collaboration: essential scientific dialogue}

Increasing globalization of the research enterprise and the need to enter into meaningful dialogues for good results make it imperative to develop local capacity. The challenge is not only to increase sound scientific production. It is to establish a pattern of collaboration that not reproduces power relations frequent in other spheres (such 
as economics) or the irrational fight against "foreign" influence (characteristic of some political discourses). A constructive contribution of Latin American science requires sound ethical oversight systems, good quality training, and awareness of the needs and expectations of the populations. Even if our trainees were not in the forefront of research, their expanded vision of the situation and the awareness of the goals of research in its proper context would help improve the translation of evidence-based facts into value-based actions

\section{Human diversity: challenge and opportunity}

Research ethics curricula face the challenge of multiethnic and multicultural contexts existent in Latin America, whose indigenous population averages $11 \%$ but varies widely from $80 \%$ in Bolivia, $60 \%$ in Guatemala and $40 \%$ in Peru. Even language diversity is a factor hampering research, particularly in the field of social studies and public health. Stigmatization has been found to affect genomics research in populations and the need for a "culture fair" approach to data gathering and interpretation is as an ethical an imperative as the need to respect dignity or request consent. The notion of "ethical sustainability", essential in our continent-wide work, stresses what international documents identify as essential in the pursuit of science at the service of development. UNESCO Universal Declaration on Bioethics and Human Rights, among other documents, underscores the vulnerability of resource-poor populations and the ethical imperative to avoid the typical "safari research": researchers from affluent societies behave as "data hunters" and disregard opportunities for real collaboration. Our emphasis on a selfgenerating faculty and permanent dialogue was an exercise in "counter-alienation" of researchers and an invitation to make real the "bi-directional" character of all ethical dialogue. The sustainability of this effort is ensured by the empirical, albeit anecdotal, evidence we have gathered during the conduction of the program. The danger of a group that is closed and immune to criticism is not supported by our experience.

These factors justify the need for highly trained professionals from developing countries to as- sume positions of leadership nationally and internationally in addressing the ethical and social issues of global health research. Since this research is inherently multidisciplinary and interdisciplinary, students from a growing range of disciplines directly or indirectly related to health seek training, thus producing a heterogeneous group whose members not necessarily share the same ethos of service or are not sufficiently committed to the relationship between science and ethics in the creation and application of knowledge. There are also wide cultural variations regarding the social implications of scientific research, the roles of researchers in the community, and the expectations regarding social impact of science and technology.

The training program developed by CIEB aimed at consolidating and expanding an "ethically sustainable" research agenda in a globalized context by training groups of professionals able to participate as researchers, policymakers, advisors to legislators, and mentors for new generations of academics. It is recognized that ethical oversight of research is best performed locally; avoiding mistrust and dependence (sometimes associated with foreign funding and foreign institutions) the autonomy of the groups and institutions was sought.

\section{Research accountability}

Research is an activity not widely performed in the Latin American continent. Scientists and scholars represent a privileged minority and many of its members feel that they belong to elites whose contribution to science and technology may be hampered or otherwise limited by ethical oversight. All along, the emphasis on accountability of researchers and research has been a permanent feature of our training effort.

\section{Approaches and experiences}

After successful application (about 30 applicants annually), five to six trainees were selected each year for courses and activities at CIEB, with an international faculty. Table 1 presents a listing of trainees with their professions, country of origin, and other relevant information. The program was 
advertised through standard academic channels (websites and flyers). Former graduates constituted a good source of information for new applicants. The selection process involved analysis of previous activities, publications and other deliverables, and support from home institutions in order to keep a position or provide development opportunities after training. The acceptance rate was on average 15 to $20 \%$, depending on the number of good applicants.

The program underwent changes. At the beginning, no virtual (on-line) component was present and trainees were hosted at the CIEB, University of Chile, for nine months; three additional months spent doing work in a country different from the country of origin. Thus, useful contribution to the development of ethics committees and ethics regulations was possible in Peru, Dominican Republic, and Costa Rica. In the ensuing years, with the introduction of virtual learning modules adapted from the CITI program developed at the University of Miami and the Fred Hutchinson Cancer Center, Seattle, Washington ${ }^{4}$, a period of virtual training was introduced before the inperson, face-to-face portion of the program. This period served for providing a common ground for all successful applicants, homogenized the language and familiarized trainees with the topics and terminology to be employed in the courses offered at CIEB in Santiago. The in-person period at CIEB was reduced to three months of full time commitment. The virtual phase served as a baseline for comparison with knowledge and attitudes at the end of the training period; it was used also as a teaching device: trainees were requested to develop modules of their own and contribute to the improvement of the virtual course.

During their stay at the CIEB in Santiago, Chile, trainees were exposed to a wide range of contents and training experiences. Courses were offered on the philosophical foundations of ethics, the history of bioethical thinking, the applications of bioethical discourse to research questions, statistical reasoning as it pertains to ethical formulation of methods, bioethical problems arising in medical and psychosocial research, qualitative and quantitative research methods, health economics,

\footnotetext{
${ }^{4}$ Available at http://wwww.citiprogram.com
}

institutionalization of bioethics, structure and functions of ethics committees, anthropology and bioethics, and other topics selected on the basis of personal interests and available teaching resources. Trainees participated in sessions of research ethics committees, had opportunity to interact with international scholars visiting the CIEB and were requested to select a topic to be dealt with in depth as a final exercise of in'person period. These essays were presented in evaluation sessions, graded, and constituted publications in accredited journals or presentations at seminars, workshops, or congresses, attendance to which was sometimes covered by special allowances from the program.

A list of publications produced by trainees and faculty during the period 2002-2011 is presented in the Appendices.

During the first two years of the program, along with formal training and delivery of the above mentioned contents, other activities were programmed. Among them was exposure to group dynamics and direct experience with emotional manifestations in group sessions. This activity demanded some effort on the part of faculty and trainees and although results were enlightening, the practice was abandoned in view of resistances voiced by some participants.

The training period at CIEB was mainly devoted to work in groups under the leadership of a faculty member, avoiding any resemblance to an asymmetrical relation of the type mentor-trainee or tutor-trainee. The covert dimension of the curriculum was to illustrate practically the dialogical nature of the bioethical enterprise and the development of bioethical discourse through open manifestation of ideas.

Among the guiding principles implemented, the following can be mentioned:

- Methodical pluralism. "Solutions" given by different disciplines to ethical dilemmas depend on methods, that is, ways of formulating, interpreting, and solving problems. The power of a profession derives from its being able to identify "real" problems and provide appropriate solutions. The teaching of sta- 
tistical methods, participant observation, qualitative and quantitative approaches to data acquisition and presentation was aimed at reinforcing the idea that no good science (in the ethical sense of good) can be obtained without careful attention to method. In addition, the multi-leveled and complex nature of scientific/ethical problems was underscored by stressing different approaches to problems.

- Perspectivism. Not to be confused with moral relativism, the presentation of different perspectives is essential in creating an adequate environment for democratic and fact-producing dialogue. This included an openness to all facts related to a problem or to decision-making, illustrating a "culture of dialogue" that is of the essence of bioethical practice in committees and commissions. Deliberation was pursued in an attempt to generate conclusions "sine ira et studio" but well grounded on argument.

- Open-mindedness. This ideal of interaction was sought after by encouraging manifestation of personal preferences and emotionladen material related to case analyses. Success was measured by requesting a personal evaluation from trainees after the completion of the face-to-face period.

- Cultural sensitiveness. The interdisciplinary nature of our effort was accompanied by the effort to underscore what is meant by a truly transcultural approach. Contact with anthropological thinking was provided by professional anthropologists who illustrated their methods and data with examples drawn from the cultural and the health fields. Their methods and approaches were considered valuable by trainees and helped to create awareness of the multiethnic and multicultural character of Latin American societies. The relevance of this knowledge for a culturally fair bioethics was stressed all along.

- Attitudinal change. Although an ambitious goal not always easy to attain, it was expected that after the training period, attitudes toward problems, ethical dilemmas, and current or possible solutions could undergo changes. Again, this was reflected in self evaluations made by trainees and faculty and was the subject matter of testimonies collected after the experience was finished.

- Improvement of communication skills. Trainees were exposed to a variety of approaches for improving written and oral communication, and special training sessions were devoted to the design and development of websites, listserv lists, slide presentations, oral reports and written materials. This was essential given the different training experiences of participants and the need to represent ideas and attitudes to wider audiences. This portion of the training experience also served to highlight moral problems associated with scientific communication and thus introduce participants to a crucial aspect of research integrity.

- Tolerance and the ability to listen. Al though implicit, the notion that a crucial competence necessary for a successful implementation of bioethical discourse is tolerance and the capacity to listen to discrepant or adversary views was reinforced throughout the training period and was continued in the aftermath of the face-to-face experience, when networking activities at the home institution were supported and followed.

When the training period was completed, participants were requested to write down formal and publishable papers and to prepare an activity in their home country or at their institution of origin, to which a faculty member was invited. Thus, continuity of effort was assured and support from the home institution assessed.

\section{Evaluation of program and outcomes}

A complete assessment of impact may require an extended time to complete and should probably consider aspects well beyond immediate effects on research ethics. Multiplying effects of the experience through the work and effort of participants probably need years to show manifest outcomes in research practice, policymaking, and committee work. These could be more qualitative than quantifiable in terms of publications, policies, or other deliverables. 
The effects of the program can be evaluated at the individual, the institutional, and the national levels.

Faculty members served as tutors and provided advice and support to trainees throughout the training period and afterwards. Evaluation consisted in written reports and feedback to participants on their degree of commitment, collaboration with the long-term goals of the program, and written productions.

Individuals participating in the program provided feedback on their perceptions and accomplishments at the end of each term and their opinions proved valuable for reformulating aims and methods. Their publications attest to a wide range of interests and issues. The evaluations made by faculty members of participation, interest, and attitude change indicate that most of the goals of the program, at the individual level, were achieved in about half the members of the teaching experience. The program itself was modified in accordance with suggestions and ideas provided by participants.

At the institutional level, the effects can be summarized in the development and implementation of training programs, ethics committees, and structure modifications. They are indicated in the appendices. An expected outcome would certainly be an improvement in the quality of research from an ethical point of view. This, however, is difficult to assess with the information available.

At the national level the effects are more difficult to estimate. They depend in part on the personal influence each trainee may have in his/her local environment and in the recognition of the importance of research ethics by officials and public.

\section{Lessons learned - the future of ethics training within the research enterprise in Latin America}

As important as written materials, in and by itself a good demonstration of success and impact is the fact that both faculty and former trainees established an enduring collaboration which re- sulted in network programs, continued support, and evaluation meetings, the last of which was held in September 2011 in Lima, Peru.

One important lesson learned from the experience was that the program did make a difference in the careers and outlook of participants. This not always found expression in their daily routines, for most of them continued performing duties and having responsibilities very similar to those previous to the learning experience. Many of them were able to install and develop ethical review committees and improve ethical oversight in their environments. In evaluation meetings, however, reports of difficulties associated with their contribution were common. This fact does not diminish the importance of this type of training but underscores the fact that the incorporation of competent human capital to the institutions depends on appropriate conditions in the countries of origin. The process of accepting bioethical practices and modes of thinking takes time. We are working in an environment of "trial and success" and "trial and error" periods. Measured by the structuring of functioning processes at trainees' home institutions or the development of regulations and activities in their countries, the program has shown different degrees of success. Individual factors and institutional variables account for different outcomes. Important has been to maintain contact and develop networking activities that reinforce the sense of leadership derived from the successful completion of the training experience. In some quarters, the support by a US institution was considered negative and criticism was voiced, suggesting that the program was a way of imposing a politically laden view of the research enterprise. Needless to say, such criticism is not worth of consideration but exists. The plain fact that the program was conceived and carried out by a Latin American institution with a representation of all views should suffice as counterargument.

There are fundamental issues uncovered by this ten-year experience with ethics of psychosocial and biomedical research in Latin America. One is the very notion of research. Standards of scholarship and university training are not uniform 
throughout the continent. This affects the way in which ethics is considered. Sometimes we had the impression that to insist on the ethical oversight of scientific practices was misguided, considering that the idea of what research really is was fuzzy or nonexistent in some contexts. In point of fact, research was confused with other activities, such as surveillance or industry-guided clinical trials; social science research projects were not considered in need of ethical evaluation due to their presumed low risk. These misunderstandings may need some time to disappear and require an in depth-analysis of cultural practices. An exploration of what research really is in different cultural settings constitutes an interesting field of enquiry. Some aspects of this cultural difference in conceptualization and of the difficulties in devising appropriate methods to tackle with ethical issues were addressed by the Program on Bioethics of the Pan American Health Organization (PAHO), a collaborative effort of the Chilean Government, the University of Chile through CIEB, and PAHO, which was operative from 1994 through $2010(3,4)$. The history and development of science funding in Latin America is a much needed enterprise and should be pursued alongside training in ethics and integrity.

Faculty members were drawn from different institutions and academic environments. It can be said that the experience was also valuable for them. Despite the fact that interactions were not always easy and that professional expertise sometimes made it difficult to interact with other members of the team and with trainees, everybody benefited from the effort in ways sometimes unexpected. The interfaces with juridical systems, knowledge of funding procedures for research, disclosure of sometimes hidden or unknown prejudices were results from the experience of interacting with other professionals or members of specific groups. Frontier researchers still mistrust ethical oversight and this may be due to a faulty implementation of it, lack of adequate development of ethics committees or vested interests. The effort is worthwhile, the project has been rewarding to carry on, and the need is still growing.

\section{A network for the advancement of a biocentric ethics}

The experience gathered throughout the years has demonstrated that an ethical approach to science and technology, in the current state of development of disciplines, by necessity must incorporate specialized knowledge, be based on deliberation and dialogue, and depend on an organic and cohesive community. This community includes researchers, policymakers, politicians, administrators, students, and lay people. Since research is a cultural process shaped by expectations, hopes, and practices, it cannot be examined isolated from other aspects of social life. In point of fact, ethical oversight of research cannot be treated independently from the "ethical level" of the community at large. Political and administrative corruption, if present in a country and accepted as normal, cannot be irrelevant for the establishment of sound scientific practices. The notion of ethical sustainability suggests that any change in attitudes, goals, and practices must be based on sound argument and endure over time.

A sustainable effort depends critically on the establishment and maintenance of communities: Epistemic communities (or cultures), Practice communities and Moral communities. These communities not necessarily overlap, although it might be expected that the moral one embraces the others and includes knowledge and its applications. A biocentric ethics is not simply another form of applied ethics. It represents a change in the paradigmatic construction of the moral universe. Not only does it go beyond the classical anthropocentrism in the formulation of moral imperatives. It is knowledge on how to produce, expand, and apply knowledge. It is also an indication that the very foundation of welfare and progress includes a joint consideration of goals and means. Goals formulated as culture and civilization. Means legitimated by discursive practices respecting persons, living beings, and environment, accepting diversity and agreeing on basic principles of communality. In order to achieve this long-term goal, adapted to the historical peculiarities of a world region, our contribution depends on dialogue and common discourses. Re- 
search ethics is just a part of culture, and culture is life in common. The establishment of a network of users of the bioethical discourse has been an important mission of CIEB and will continue to be in the future. Thanks are to be expressed to the institutions that make it possible (especially the University of Chile) and to the funders (NIHFogarty, the Alexander von Humboldt Foundation, among others).

\section{References}

1. Lolas F. Bioética en la Universidad de Chile. Anales de la Universidad de Chile, Sexta Serie 1998; 8: 13-18.

2. Horng S, Grady C. Misunderstanding in Clinical Research: Distinguishing Therapeutic Misconception, Therapeutic Misestimating, \& Therapeutic Optimism. IRB: Ethics and Human Research (Jan-Feb.) 2003; 25(1): 11-16

3. Lolas F. Bioética en América Latina. Una década de evolución. Monografía de Acta Bioethica $\mathrm{N}^{\circ} 4$. Santiago de Chile: CIEB Universidad de Chile; 2010.

4. Lolas F. (editor) Diálogo y cooperación en salud. Diez años de bioética en OPS. Santiago de Chile: Programa de Bioética OPS/OMS; 2004.

Received: December 20, 2011

Accepted: January 10, 2012 


\section{APPENDICES}

\section{CIEB PROGRAM NIH-FOGARTY Grant R25TW006056}

Table 1: CIEB Fogarty Trainees 2003-2011

\begin{tabular}{|c|c|c|c|}
\hline Countries & Professions & Characteristics & Contributions \\
\hline $\begin{array}{l}\text { Mexico }-8 \\
\text { Chile }-7 \\
\text { Colombia }-7 \\
\text { Argentina }-6 \\
\text { Peru - } 5 \\
\text { Nicaragua - } 3 \\
\text { Ecuador - } 3 \\
\text { Bolivia - } 2 \\
\text { Honduras - } 2 \\
\text { El Salvador - } 1 \\
\text { Brazil - } 1 \\
\text { Uruguay - } 1 \\
\text { Dominican Republic }-1 \\
\text { Guatemala - } 1 \\
\text { Spain - } 1\end{array}$ & $\begin{array}{l}\text { Medicine }-18 \\
\text { Dentist }-8 \\
\text { Lawyer }-4 \\
\text { Pharmacy }-3 \\
\text { Chemistry }-3 \\
\text { Nurse }-2 \\
\text { Sociology }-2 \\
\text { Philosophy - } 2 \\
\text { Microbiology - } 2 \\
\text { Obstetrician - } 1 \\
\text { Biology - } 1 \\
\text { Veterinary - } 1 \\
\text { International Relations }-1 \\
\text { Epidemiology - } 1 \\
\text { Psychology - } 1\end{array}$ & $\begin{array}{l}\text { Number: } 50 \\
\text { Masculine: } 16 \\
\text { Feminine: } 34\end{array}$ & $\begin{array}{l}\text { Program Research Projects: } 50 \\
\text { Additional Research Projects: } 14 \\
\text { Publications } \\
\text { - Articles: } 54 \\
\text { - Books, chapters: } 52 \\
\text { - Web: } 23 \\
\text { - Online Virtual Modules: } 6 \\
\text { - Web Bulletins: } 3 \\
\text { - Web Sites: } 3 \\
\text { Health Research Policies and Regulations: } 26 \\
\text { Country ethics of research workshop } \\
\text { interventions: } 22 \\
\text { Development of Training programs in ethics } \\
\text { of research: } 20 \\
\text { Creation of new Scientific Ethical Review } \\
\text { Committees: } 9 \\
\text { Participation in Scientific Ethical Review } \\
\text { Committees: } 28 \\
\text { Participation in National Bioethics } \\
\text { Commissions: } 6 \\
\text { Honors: } 7 \\
\text { Presentations at Conferences: } 104\end{array}$ \\
\hline
\end{tabular}


Table 2: Long-Term Trainees Interdisciplinary Center for Studies on Bioethics, University of Chile Fogarty Project who completed 1 year training on ethics of biomedical and psychosocial research (including coursework, research and practice, diploma degree conferred).

\begin{tabular}{|c|c|c|c|c|c|}
\hline Name & $\begin{array}{l}\text { Country of } \\
\text { Origin }\end{array}$ & Degree & $\begin{array}{l}\text { Period of Trai- } \\
\text { ning }\end{array}$ & $\begin{array}{l}\text { Research project } \\
\text { during training }\end{array}$ & Institution \\
\hline Luis Moreno & Peru & BS Chemist & $2003-2004$ & $\begin{array}{l}\text { Ethical Analysis } \\
\text { of Bioavailabili- } \\
\text { ty and Bioequi- } \\
\text { valence Studies } \\
\text { of Pharmaceuti- } \\
\text { cal Products } \\
\text { Contain in } \\
\text { Latin America } \\
\text { Legislations }\end{array}$ & $\begin{array}{l}\text { National Insti- } \\
\text { tute of Health } \\
\text { (Quality Con- } \\
\text { trol). Pharmacy } \\
\text { Dept. Major } \\
\text { University San } \\
\text { Marcos, Lima, } \\
\text { Peru }\end{array}$ \\
\hline Agustin Estevez & Argentina & BA Philosophy & $2003-2004$ & $\begin{array}{l}\text { The Role of } \\
\text { Lay Civilians } \\
\text { in Scientific } \\
\text { Ethical Review } \\
\text { Committees, } \\
\text { Guidelines } \\
\text { for the De- } \\
\text { velopment } \\
\text { of Scientific } \\
\text { Ethical Review } \\
\text { Committees }\end{array}$ & $\begin{array}{l}\text { South Natio- } \\
\text { nal University, } \\
\text { Argentina }\end{array}$ \\
\hline $\begin{array}{l}\text { Lautaro Fernan- } \\
\text { dez }\end{array}$ & Chile & BS Obstetrics & $2003-2004$ & $\begin{array}{l}\text { Degree of } \\
\text { legibility of In- } \\
\text { formed Consent } \\
\text { Protocols for } \\
\text { Research Invol- } \\
\text { ving Human } \\
\text { Beings Appro- } \\
\text { ved by Scientific } \\
\text { Ethical Review } \\
\text { Committees at } \\
\text { Health Services } \\
\text { in Chile }\end{array}$ & $\begin{array}{l}\text { South Metro- } \\
\text { politan Health } \\
\text { Service, Santia- } \\
\text { go, Chile }\end{array}$ \\
\hline Cesar Lara & Mexico & BA Law & $2003-2004$ & $\begin{array}{l}\text { Legal Aspects } \\
\text { for Ethical } \\
\text { Review of Re- } \\
\text { search Protocols }\end{array}$ & $\begin{array}{l}\text { Ministry of } \\
\text { Health, Mexico }\end{array}$ \\
\hline $\begin{array}{l}\text { Katya Rodri- } \\
\text { guez }\end{array}$ & Ecuador & M.D & $2003-2004$ & $\begin{array}{l}\text { Ethics of Place- } \\
\text { bo of Biome- } \\
\text { dical Research } \\
\text { Performed at } \\
\text { Chile }\end{array}$ & $\begin{array}{l}\text { Catholic Uni- } \\
\text { versity, Quito, } \\
\text { Ecuador }\end{array}$ \\
\hline
\end{tabular}


Ethics in psychosocial and biomedical research - Fernando Lolas, Eduardo Rodriguez

\begin{tabular}{|c|c|c|c|c|c|}
\hline Name & $\begin{array}{l}\text { Country of } \\
\text { Origin }\end{array}$ & Degree & $\begin{array}{l}\text { Period of Trai- } \\
\text { ning }\end{array}$ & $\begin{array}{l}\text { Research project } \\
\text { during training }\end{array}$ & Institution \\
\hline $\begin{array}{l}\text { Monica Her- } \\
\text { nandez }\end{array}$ & Chile & BS Nursing & $2003-2004$ & $\begin{array}{l}\text { Role of Subjects } \\
\text { in Biomedical } \\
\text { Research }\end{array}$ & $\begin{array}{l}\text { Center for Va- } \\
\text { ccine Develop- } \\
\text { ment, Santiago, } \\
\text { Chile }\end{array}$ \\
\hline Alexandre Bota & Spain & Ph.D. & $2003-2004$ & $\begin{array}{l}\text { Biological Sam- } \\
\text { ples Ownership }\end{array}$ & $\begin{array}{l}\text { Institute Tec- } \\
\text { noetica, Barce- } \\
\text { lona, Spain }\end{array}$ \\
\hline Adrana Hevia & Chile & BA Sociology & $2003-2004$ & $\begin{array}{l}\text { AIDS Patients } \\
\text { Motivations as } \\
\text { Participants in } \\
\text { Research }\end{array}$ & $\begin{array}{l}\text { University of } \\
\text { Santiago, Chile }\end{array}$ \\
\hline $\begin{array}{l}\text { Ana Laura } \\
\text { Buendia }\end{array}$ & Mexico & D.D.S. & 2004-2005 & $\begin{array}{l}\text { Analysis of } \\
\text { how ethics is } \\
\text { taught at dental } \\
\text { schools in } \\
\text { U.S.A. }\end{array}$ & $\begin{array}{l}\text { University of } \\
\text { Southern Cali- } \\
\text { fornia, School } \\
\text { of Dentistry }\end{array}$ \\
\hline Pedro Jimenez & Mexico & $\begin{array}{l}\text { Ph.D. Chemis- } \\
\text { try }\end{array}$ & 2004-2005 & $\begin{array}{l}\text { How the infor- } \\
\text { med consent } \\
\text { of patients is } \\
\text { affected by the } \\
\text { use of biologi- } \\
\text { cal samples for } \\
\text { research after } \\
\text { diagnosis in } \\
\text { clinical labs at } \\
\text { Santiago, Chile } \\
\text { and in Santo } \\
\text { Domingo }\end{array}$ & $\begin{array}{l}\text { Social Security } \\
\text { Mexican Insti- } \\
\text { tute, Mexico }\end{array}$ \\
\hline Jorge A. Alvarez & Mexico & M.D. & 2004-2005 & $\begin{array}{l}\text { Types of Anxie- } \\
\text { ty in couples } \\
\text { who participate } \\
\text { in assisted } \\
\text { reproduction } \\
\text { proceedings } \\
\text { for the issue of } \\
\text { donating their } \\
\text { embryos for } \\
\text { research in the } \\
\text { clinical hospital } \\
\text { of the Universi- } \\
\text { ty of Chile and } \\
\text { in Lima, Peru }\end{array}$ & $\begin{array}{l}\text { Social Security } \\
\text { Mexican Insti- } \\
\text { tute, Mexico }\end{array}$ \\
\hline
\end{tabular}




\begin{tabular}{|c|c|c|c|c|c|}
\hline Name & $\begin{array}{l}\text { Country of } \\
\text { Origin }\end{array}$ & Degree & $\begin{array}{l}\text { Period of Trai- } \\
\text { ning }\end{array}$ & $\begin{array}{l}\text { Research project } \\
\text { during training }\end{array}$ & Institution \\
\hline $\begin{array}{l}\text { Gabriel J. Mon- } \\
\text { toya }\end{array}$ & Colombia & $\begin{array}{l}\text { M.D. Psychia- } \\
\text { try }\end{array}$ & $2004-2005$ & $\begin{array}{l}\text { Limits in the } \\
\text { confidentiality } \\
\text { of patient's } \\
\text { sexual orienta- } \\
\text { tion assigned by } \\
\text { psychiatrists }\end{array}$ & $\begin{array}{l}\text { University of } \\
\text { Antioquia, } \\
\text { Medellin, Co- } \\
\text { lombia }\end{array}$ \\
\hline Maria A. Torres & Chile & D.D.S. & $2004-2005$ & $\begin{array}{l}\text { Oral Health Re- } \\
\text { search Bioethics }\end{array}$ & $\begin{array}{l}\text { Faculty of Den- } \\
\text { tistry Universi- } \\
\text { ty of Chile }\end{array}$ \\
\hline $\begin{array}{l}\text { Carmen A. } \\
\text { Cardozo }\end{array}$ & Colombia & D.D.S. & $2005-2006$ & $\begin{array}{l}\text { The principle } \\
\text { of the three } \\
\text { R's expressing } \\
\text { values and } \\
\text { virtues. Strategy } \\
\text { for quality and } \\
\text { competitiveness } \\
\text { in research in- } \\
\text { volving experi- } \\
\text { mental animals } \\
\text { at Colombia } \\
\text { and Chile }\end{array}$ & $\begin{array}{l}\text { Biotechnology } \\
\text { Institute and } \\
\text { Dept. of Basic } \\
\text { Sciences Natio- } \\
\text { nal University } \\
\text { of Colombia }\end{array}$ \\
\hline $\begin{array}{l}\text { Melba de la } \\
\text { Cruz Barrantes }\end{array}$ & Nicaragua & M.D. & $2005-2006$ & $\begin{array}{l}\text { Main ethical } \\
\text { dilemmas for } \\
\text { family and me- } \\
\text { dical personnel } \\
\text { in treatment } \\
\text { decision ma- } \\
\text { king for acute } \\
\text { symptoms in } \\
\text { severe Alzhei- } \\
\text { mer patients }\end{array}$ & $\begin{array}{l}\text { Drugs Dept. } \\
\text { Center for Me- } \\
\text { dical Informa- } \\
\text { tion, Regulation } \\
\text { Dept. Ministry } \\
\text { of Health, Nica- } \\
\text { ragua }\end{array}$ \\
\hline $\begin{array}{l}\text { Liliana Mon- } \\
\text { dragon }\end{array}$ & Mexico & $\begin{array}{l}\text { Ph.D. Psycho- } \\
\text { logy }\end{array}$ & $2005-2006$ & $\begin{array}{l}\text { Ethics of } \\
\text { psychology: } \\
\text { bioethical } \\
\text { dilemmas for } \\
\text { therapeutic } \\
\text { approaching } \\
\text { towards suicidal } \\
\text { attempts }\end{array}$ & $\begin{array}{l}\text { Epidemiology } \\
\text { and Psychoso- } \\
\text { cial Dept. } \\
\text { National Insti- } \\
\text { tute of Psychia- } \\
\text { try Ramon de la } \\
\text { Fuente Muñiz, } \\
\text { Mexico }\end{array}$ \\
\hline $\begin{array}{l}\text { Melvy Ormae- } \\
\text { che }\end{array}$ & Peru & BS Nursing & $2005-2006$ & $\begin{array}{l}\text { Informed con- } \\
\text { sent for research } \\
\text { on indigenous } \\
\text { populations }\end{array}$ & $\begin{array}{l}\text { Epidemiology } \\
\text { Dept. Minis- } \\
\text { try of Health, } \\
\text { Lima, Peru }\end{array}$ \\
\hline Margot Vidal & Peru & $\begin{array}{l}\text { M.D. Epide- } \\
\text { miology }\end{array}$ & $2005-2006$ & $\begin{array}{l}\text { Importance } \\
\text { of social and } \\
\text { family support } \\
\text { for treatment } \\
\text { adherence in } \\
\text { HIV/AIDS } \\
\text { patients }\end{array}$ & $\begin{array}{l}\text { Cayetano He- } \\
\text { redia Peruvian } \\
\text { University, } \\
\text { Lima, Peru }\end{array}$ \\
\hline
\end{tabular}




\begin{tabular}{|c|c|c|c|c|c|}
\hline Name & $\begin{array}{l}\text { Country of } \\
\text { Origin }\end{array}$ & Degree & $\begin{array}{l}\text { Period of Trai- } \\
\text { ning }\end{array}$ & $\begin{array}{l}\text { Research project } \\
\text { during training }\end{array}$ & Institution \\
\hline Blanca Borquez & Chile & BA Law & $2005-2006$ & $\begin{array}{l}\text { Informed con- } \\
\text { sent in adoles- } \\
\text { cents }\end{array}$ & $\begin{array}{l}\text { EJE Salud } \\
\text { LTDA Library } \\
\text { Chilean Con- } \\
\text { gress }\end{array}$ \\
\hline $\begin{array}{l}\text { Elizabeth Mer- } \\
\text { cado }\end{array}$ & Nicaragua & M.D. & $2006-2007$ & $\begin{array}{l}\text { Euthanasia and } \\
\text { extreme measu- } \\
\text { res in patients } \\
\text { with bad prog- } \\
\text { nosis and health } \\
\text { priorities }\end{array}$ & $\begin{array}{l}\text { National Insti- } \\
\text { tute for Medical } \\
\text { Sciences \& } \\
\text { Nutrition, } \\
\text { Nicaragua. } \\
\text { Hospital Dr. } \\
\text { Roberto Cal- } \\
\text { deron }\end{array}$ \\
\hline Saul Diaz & El Salvador & M.D. & $2006-2007$ & $\begin{array}{l}\text { Retrospecti- } \\
\text { ve study on } \\
\text { the impact of } \\
\text { bioethics in the- } \\
\text { sis of medical } \\
\text { students at the } \\
\text { university of } \\
\text { Chile }\end{array}$ & $\begin{array}{l}\text { Research Center } \\
\text { Dept. of medi- } \\
\text { cine University } \\
\text { of El Salvador }\end{array}$ \\
\hline Doris E. Ospina & Colombia & $\begin{array}{l}\text { Ph.D. Psycho- } \\
\text { logy }\end{array}$ & $2006-2007$ & $\begin{array}{l}\text { Keeping com- } \\
\text { pany to patients } \\
\text { with bipolar } \\
\text { disorder: Con- } \\
\text { tributions by } \\
\text { Psycho Educa- } \\
\text { tion } \\
\end{array}$ & $\begin{array}{l}\text { Nursery School } \\
\text { University of } \\
\text { Antioquia, } \\
\text { Colombia }\end{array}$ \\
\hline $\begin{array}{l}\text { Alma E. Ren- } \\
\text { don }\end{array}$ & Mexico & $\begin{array}{l}\text { BS Internatio- } \\
\text { nal Relations }\end{array}$ & $2006-2007$ & $\begin{array}{l}\text { Patents in } \\
\text { biotechnolo- } \\
\text { gy for health: } \\
\text { Perception and } \\
\text { tendencies at } \\
\text { Latin America }\end{array}$ & $\begin{array}{l}\text { Ministry of } \\
\text { Health, Mexico }\end{array}$ \\
\hline $\begin{array}{l}\text { Armando Ulloa } \\
\text { Gonzalez }\end{array}$ & Nicaragua & M.D. & $2007-2008$ & $\begin{array}{l}\text { Informed Con- } \\
\text { sent in Hospi- } \\
\text { tals at Managua, } \\
\text { Nicaragua } \\
\end{array}$ & $\begin{array}{l}\text { National Auto- } \\
\text { nomous Univer- } \\
\text { sity, Nicaragua }\end{array}$ \\
\hline $\begin{array}{l}\text { Catalina Beltran } \\
\text { Sarmiento }\end{array}$ & Colombia & M.D. & $2007-2008$ & $\begin{array}{l}\text { Ethical im- } \\
\text { plications of } \\
\text { the destiny of } \\
\text { human embryos } \\
\text { leftover by in vi- } \\
\text { tro fertilization } \\
\text { from a legal } \\
\text { and economical } \\
\text { context }\end{array}$ & $\begin{array}{l}\text { National } \\
\text { University of } \\
\text { Colombia } \\
\text { MSD Pharma- } \\
\text { ceutic }\end{array}$ \\
\hline
\end{tabular}




\begin{tabular}{|c|c|c|c|c|c|}
\hline Name & $\begin{array}{l}\text { Country of } \\
\text { Origin }\end{array}$ & Degree & $\begin{array}{l}\text { Period of Trai- } \\
\text { ning }\end{array}$ & $\begin{array}{l}\text { Research project } \\
\text { during training }\end{array}$ & Institution \\
\hline $\begin{array}{l}\text { Elizabeth C. } \\
\text { Zwareva }\end{array}$ & Bolivia & BS Nursing & $2007-2008$ & $\begin{array}{l}\text { Bioethics incor- } \\
\text { poration in the } \\
\text { curricula of the } \\
\text { Bolivian Catho- } \\
\text { lic University }\end{array}$ & $\begin{array}{l}\text { Catholic Uni- } \\
\text { versity, Bolivia }\end{array}$ \\
\hline $\begin{array}{l}\text { Carmen R. Gar- } \\
\text { cia Rupaya }\end{array}$ & Peru & D.D.S. & $2007-2008$ & $\begin{array}{l}\text { Construction, } \\
\text { validation and } \\
\text { application of } \\
\text { an instrument } \\
\text { to measure } \\
\text { dentistry stu- } \\
\text { dents attitu- } \\
\text { des towards } \\
\text { bioethics }\end{array}$ & $\begin{array}{l}\text { Cayetano He- } \\
\text { redia Peruvian } \\
\text { University, } \\
\text { Lima, Peru }\end{array}$ \\
\hline $\begin{array}{l}\text { Gilberto Gam- } \\
\text { boa Bernal }\end{array}$ & Colombia & M.D. & $2007-2008$ & $\begin{array}{l}\text { Quality of Life } \\
\text { in Clinical } \\
\text { Practice }\end{array}$ & $\begin{array}{l}\text { University La } \\
\text { Sabana, Colom- } \\
\text { bia }\end{array}$ \\
\hline $\begin{array}{l}\text { Carlos Briones } \\
\text { Reyes }\end{array}$ & Chile & $\begin{array}{l}\text { BS } \\
\text { Veterinary }\end{array}$ & 2008-2009 & $\begin{array}{l}\text { Description of } \\
\text { ethical thinking } \\
\text { of organizations } \\
\text { that work with } \\
\text { pets in Santia- } \\
\text { go, Chile, with } \\
\text { respect to their } \\
\text { moral status } \\
\text { and euthanasia } \\
\text { control mea- } \\
\text { sures }\end{array}$ & $\begin{array}{l}\text { Veterinary } \\
\text { Clinic Nueva } \\
\text { Renca, Santia- } \\
\text { go, Chile } \\
\text { University of } \\
\text { Chile }\end{array}$ \\
\hline $\begin{array}{l}\text { Maria Rita } \\
\text { Carvalho Garbi- } \\
\text { Novaes }\end{array}$ & Brazil & $\begin{array}{l}\text { Ph.D. Phar- } \\
\text { macy }\end{array}$ & 2008-2009 & $\begin{array}{l}\text { Brazilian } \\
\text { diagnosis of } \\
\text { ethical and } \\
\text { bioethical issues } \\
\text { insertion into } \\
\text { the curricula of } \\
\text { medicine }\end{array}$ & $\begin{array}{l}\text { Health Secre- } \\
\text { tary Federal } \\
\text { District, FE- } \\
\text { PECS/SES-DF, } \\
\text { Brasilia, Brazil }\end{array}$ \\
\hline $\begin{array}{l}\text { Juan Jose Ore- } \\
\text { llana Arauco }\end{array}$ & Bolivia & D.D.S. & $2008-2009$ & $\begin{array}{l}\text { Informed con- } \\
\text { sent procedures } \\
\text { in dentristy } \\
\text { practice at } \\
\text { Private Tech- } \\
\text { nical Univer- } \\
\text { sity Cosmos } \\
\text { (UNITEPC), } \\
\text { Cochabamba }\end{array}$ & $\begin{array}{l}\text { Central Uni- } \\
\text { versity, Private } \\
\text { Technical Cos- } \\
\text { mos University, } \\
\text { Cochabamba, } \\
\text { Bolivia }\end{array}$ \\
\hline $\begin{array}{l}\text { Carlos Walter } \\
\text { Sanabria Ortega }\end{array}$ & Paraguay & BA Philosophy & $2008-2009$ & $\begin{array}{l}\text { Background, } \\
\text { justification } \\
\text { and norms for } \\
\text { the creation } \\
\text { of a National } \\
\text { Scientific Ethics } \\
\text { Board at Para- } \\
\text { guay }\end{array}$ & $\begin{array}{l}\text { National Uni- } \\
\text { versity of Asun- } \\
\text { cion, Paraguay }\end{array}$ \\
\hline
\end{tabular}




\begin{tabular}{|c|c|c|c|c|c|}
\hline Name & $\begin{array}{l}\text { Country of } \\
\text { Origin }\end{array}$ & Degree & $\begin{array}{l}\text { Period of Trai- } \\
\text { ning }\end{array}$ & $\begin{array}{l}\text { Research project } \\
\text { during training }\end{array}$ & Institution \\
\hline $\begin{array}{l}\text { Hugo Rodri- } \\
\text { guez Almada }\end{array}$ & Uruguay & M.D. & 2008-2009 & $\begin{array}{l}\text { Informed Con- } \\
\text { sent in research } \\
\text { in Uruguayan } \\
\text { legal context }\end{array}$ & $\begin{array}{l}\text { University La } \\
\text { Republica, } \\
\text { Uruguay }\end{array}$ \\
\hline $\begin{array}{l}\text { Vilma E. Espi- } \\
\text { noza }\end{array}$ & Honduras & $\begin{array}{l}\text { MS Microbiolo- } \\
\text { gy and Clinical } \\
\text { Chemistry }\end{array}$ & 2008-2009 & $\begin{array}{l}\text { Diagnosis of } \\
\text { ethics of re- } \\
\text { search at Hon- } \\
\text { duras: Norms } \\
\text { and functioning } \\
\text { of scientific } \\
\text { ethical review } \\
\text { committees }\end{array}$ & $\begin{array}{l}\text { National Auto- } \\
\text { nomous Univer- } \\
\text { sity, Honduras }\end{array}$ \\
\hline $\begin{array}{l}\text { Pablo A. Melo- } \\
\text { nari }\end{array}$ & Argentina & M.D. & 2008-2009 & \begin{tabular}{|l|} 
Diagnosis of \\
Bioethical \\
concerns in \\
Clinical Practice \\
Hospital Notti \\
(Mendoza, \\
Argentina)
\end{tabular} & $\begin{array}{l}\text { National Uni- } \\
\text { versity of Cuyo } \\
\text { Pediatric } \\
\text { Hospital Notti, } \\
\text { Mendoza, } \\
\text { Argentina }\end{array}$ \\
\hline $\begin{array}{l}\text { Hilda Valencia } \\
\text { Marroquin de } \\
\text { Abril }\end{array}$ & Guatemala & D.D.S. & 2009-2010 & \begin{tabular}{|l|} 
Level of ethics \\
of research \\
competence \\
of research \\
projects, Re- \\
search Division, \\
University San \\
Carlos, Guate- \\
mala \\
\end{tabular} & $\begin{array}{l}\text { University San } \\
\text { Carlos Guate- } \\
\text { mala }\end{array}$ \\
\hline $\begin{array}{l}\text { Lilian Patricia } \\
\text { Moncayo Yerovi }\end{array}$ & Ecuador & D.D.S. & 2009-2010 & $\begin{array}{l}\text { Diagnosis of } \\
\text { human subjects } \\
\text { protection in } \\
\text { norms and } \\
\text { regulations at } \\
\text { Ecuador }\end{array}$ & $\begin{array}{l}\text { Ministry of } \\
\text { Health Ecuador }\end{array}$ \\
\hline $\begin{array}{l}\text { Elsa Diaz San- } \\
\text { tana }\end{array}$ & $\begin{array}{l}\text { Dominican } \\
\text { Republic }\end{array}$ & $\begin{array}{l}\text { BS Pharmacy } \\
\text { MS Bioethics }\end{array}$ & 2009-2010 & $\begin{array}{l}\text { Ethical basis for } \\
\text { fulfilling Hos- } \\
\text { pital Pharma- } \\
\text { ceutical Basic } \\
\text { Norms at Hos- } \\
\text { pital Doctor } \\
\text { Dario Contre- } \\
\text { ras, Pharmacy } \\
\text { Service, Santo } \\
\text { Domingo, } \\
\text { Dominican } \\
\text { Republic }\end{array}$ & $\begin{array}{l}\text { Hospital } \\
\text { Doctor Dario } \\
\text { Contreras, } \\
\text { Santo Domin- } \\
\text { go, Dominican } \\
\text { Republic }\end{array}$ \\
\hline $\begin{array}{l}\text { Aida Rozxana } \\
\text { Merlo Romero }\end{array}$ & Honduras & M.D. & 2009-2010 & $\begin{array}{l}\text { Diagnosis of } \\
\text { research ethics } \\
\text { at National } \\
\text { Autonomous } \\
\text { University, Valle } \\
\text { Sula, Honduras }\end{array}$ & $\begin{array}{l}\text { National } \\
\text { Autonomous } \\
\text { University Valle } \\
\text { de Sula, Hon- } \\
\text { duras }\end{array}$ \\
\hline
\end{tabular}




\begin{tabular}{|c|c|c|c|c|c|}
\hline Name & $\begin{array}{l}\text { Country of } \\
\text { Origin }\end{array}$ & Degree & $\begin{array}{l}\text { Period of Trai- } \\
\text { ning }\end{array}$ & $\begin{array}{l}\text { Research project } \\
\text { during training }\end{array}$ & Institution \\
\hline $\begin{array}{l}\text { Liliana Edith } \\
\text { Caballero }\end{array}$ & Argentina & M.D. & $2009-2010$ & $\begin{array}{l}\text { Monitoring of } \\
\text { clinical trials for } \\
\text { drug approval } \\
\text { during trial } \\
\text { execution }\end{array}$ & $\begin{array}{l}\text { Kennedy Uni- } \\
\text { versity, Buenos } \\
\text { Aires, Argentina }\end{array}$ \\
\hline $\begin{array}{l}\text { Veronica Nieto } \\
\text { Canessa }\end{array}$ & Uruguay & M.D. & $2010-2011$ & $\begin{array}{l}\text { Norms related } \\
\text { to care for } \\
\text { handicaps. } \\
\text { A bioethical } \\
\text { reflection }\end{array}$ & $\begin{array}{l}\text { University La } \\
\text { Republica, } \\
\text { Uruguay }\end{array}$ \\
\hline $\begin{array}{l}\text { Julieta Ivone } \\
\text { Castro }\end{array}$ & Mexico & $\begin{array}{l}\text { M.D. } \\
\text { PhD Biomedi- } \\
\text { cal Sciences }\end{array}$ & $2010-2011$ & $\begin{array}{l}\text { Bioethical } \\
\text { analysis of el- } \\
\text { derly health care }\end{array}$ & $\begin{array}{l}\text { National Insti- } \\
\text { tute of Public } \\
\text { Health, Mexico }\end{array}$ \\
\hline $\begin{array}{l}\text { Carmen Isolina } \\
\text { Ayala Jara }\end{array}$ & Peru & $\begin{array}{l}\text { M.D. } \\
\text { PhD Pharmacy } \\
\text { Chemistry }\end{array}$ & $2010-2011$ & $\begin{array}{l}\text { Ethical analy- } \\
\text { sis of research } \\
\text { thesis involving } \\
\text { human beings } \\
\text { at National } \\
\text { University of } \\
\text { Trujillo }\end{array}$ & $\begin{array}{l}\text { National } \\
\text { University of } \\
\text { Trujillo, Peru }\end{array}$ \\
\hline Sandra Pavon & Argentina & M.D. & $2010-2011$ & $\begin{array}{l}\text { Influence in } \\
\text { society of sleep } \\
\text { alterations. } \\
\text { Bioethical } \\
\text { reflection }\end{array}$ & $\begin{array}{l}\text { Hospital Diego } \\
\text { Paroissen, Ar- } \\
\text { gentina }\end{array}$ \\
\hline $\begin{array}{l}\text { Martha Lucia } \\
\text { Rodriguez Paz }\end{array}$ & Colombia & D.D.S. & $2010-2011$ & $\begin{array}{l}\text { Analysis of } \\
\text { bioethics } \\
\text { knowledge by } \\
\text { students at } \\
\text { Health Dept., } \\
\text { University El } \\
\text { Valle, Cali, } \\
\text { Colombia }\end{array}$ & $\begin{array}{l}\text { University El } \\
\text { Valle, Cali, } \\
\text { Colombia }\end{array}$ \\
\hline $\begin{array}{l}\text { Olga del Car- } \\
\text { men Maldo- } \\
\text { nado }\end{array}$ & Colombia & $\begin{array}{l}\text { MA Philosoph, } \\
\text { Sociology } \\
\text { MS Bioethics }\end{array}$ & 2011-2012 & $\begin{array}{l}\text { Perception of } \\
\text { the importance } \\
\text { of bioethics for } \\
\text { public policies } \\
\text { management } \\
\text { oriented to pre- } \\
\text { vention of child } \\
\text { abuse in Bogo- } \\
\text { ta, Colombia }\end{array}$ & $\begin{array}{l}\text { Javeriana Uni- } \\
\text { versity, Bogota, } \\
\text { Colombia }\end{array}$ \\
\hline $\begin{array}{l}\text { Yuri Jesus Mari- } \\
\text { mon Diaz }\end{array}$ & Ecuador & M.D. & 2011-2012 & $\begin{array}{l}\text { Public policies } \\
\text { as mechanism } \\
\text { for the protec- } \\
\text { tion of HIV/ } \\
\text { AIDS patients } \\
\text { research subjects } \\
\text { in Ecuador }\end{array}$ & $\begin{array}{l}\text { Ministry of } \\
\text { Health, Ecua- } \\
\text { dor }\end{array}$ \\
\hline
\end{tabular}


Ethics in psychosocial and biomedical research - Fernando Lolas, Eduardo Rodriguez

\begin{tabular}{|l|l|l|l|l|l|}
\hline Name & $\begin{array}{l}\text { Country of } \\
\text { Origin }\end{array}$ & Degree & $\begin{array}{l}\text { Period of Trai- } \\
\text { ning }\end{array}$ & $\begin{array}{l}\text { Research project } \\
\text { during training }\end{array}$ & Institution \\
\hline $\begin{array}{l}\text { Miriam Mag- } \\
\text { dalena Sanders } \\
\text { Bruletti }\end{array}$ & Argentina & BA Law & $2011-2012$ & $\begin{array}{l}\text { Level of } \\
\text { knowledge } \\
\text { about ethics } \\
\text { of research } \\
\text { guidelines of } \\
\text { law students in } \\
\text { a private uni- } \\
\text { versity, Buenos } \\
\text { Aires, Argentina }\end{array}$ & $\begin{array}{l}\text { University of } \\
\text { Flores, Buenos } \\
\text { Aires, Argentina }\end{array}$ \\
\hline $\begin{array}{l}\text { Maria Hilda } \\
\text { Garcia Perez }\end{array}$ & Mexico & $\begin{array}{l}\text { Ph.D Epide- } \\
\text { miology }\end{array}$ & $2011-2012$ & $\begin{array}{l}\text { Public policies, } \\
\text { health care } \\
\text { satisfaction } \\
\text { and indigenous } \\
\text { autonomy: The } \\
\text { case of Oppor- } \\
\text { tunity Program, } \\
\text { Mexican-US } \\
\text { Border }\end{array}$ & $\begin{array}{l}\text { Arizona State } \\
\text { University }\end{array}$ \\
\hline $\begin{array}{l}\text { Matalia Paola } \\
\text { Cosmai }\end{array}$ & Argentina & M.D. & $2011-2012$ & $\begin{array}{l}\text { Perception } \\
\text { of informed } \\
\text { consent process } \\
\text { by physicians } \\
\text { participating in } \\
\text { research studies }\end{array}$ & $\begin{array}{l}\text { University of } \\
\text { Auenos Aires, }\end{array}$ \\
\hline
\end{tabular}




\section{APPENDIX I}

\section{Publications of trainees}

\section{Articles in peer reviewed scientific journals}

1. Aguilera RM, Mondragon LB, Medina Mora ME. Consideraciones eticas en intervenciones comunitarias: la pertinencia del consentimiento informado. Revista de Salud Mental 2008; 31(2): 129-138.

2. Alfaro Carballido D, Garcia CR. Percepcion del proceso de consentimiento informado en pacientes de la Clinica Estomatologica Central de la Universidad Peruana Cayetano Heredia. Revista Estomatologica Herediana 2011; 21(1): 5-12.

3. Alvarez JA. El estatus del embrion humano desde el gradualismo. Gaceta Medica de Mexico 2007; 143(3): 267-277.

4. Alvarez JA. Historia contemporanea: las tecnicas complejas de reproduccion asistida. Ginecologia y Obstetricia de Mexico 2007; 75(3): 293-302.

5. Alvarez JA. Historia contemporanea: las tecnicas complejas de reproduccion asistida. Ginecologia y Obstetricia de Mexico 2007; 75(3): 293-302.

6. Alvarez JA. La objecion de conciencia: el marco mexicano. Anales Facultad de Medicina Lima 2007; 68(1): 80-86.

7. Alvarez JA. Mecanismo de la fecundacion humana. Ginecologia y Obstetricia (Peru) 2007; 53(1): 45-52.

8. Alvarez JA. Politicas publicas e investigacion en embriones humanos. Revista Facultad de Medicina UNAM 2005; 48(6): 242-247.

9. Alvarez JA. Sexualidad en parejas con problemas de fertilidad. Gaceta Medica de Mexico 2007; 143(1): 65-71.

10. Alvarez JA. Tipos de ansiedad de las parejas tratadas con fertilizacion asistida frente a la donacion hipotetica de embriones en el Hospital Clinico de la Universidad de Chile. Ginecologia y Obstetricia de Mexico 2005; 73: 163-172.

11. Alvarez JA. Tipos de ansiedad de parejas tratadas con fecundacion asistida frente a la donacion hipotetica de embriones para investigacion en una clinica privada en Lima, Peru. Gaceta Medica de Mexico 2006; 142(3): 223-228.

12. Alvarez JA. Tipos de ansiedad frente a la donacion hipotetica de embriones para investigacion. Una aproximacion desde la perspectiva de genero. Salud Mental 2006; 29(5): 59-65.

13. Alvarez, JA. Comentarios sobre causales de despenalizacion entre estudiantes de pregrado de la facultad de medicina de la Universidad Autonoma de Ciudad Juarez (UACJ) ante la interrupcion voluntaria del embarazo (IVE). Revista de Investigacion Clinica 2007; 59(5): 348-355.

14. Alvarez, JA. La controversia sobre la viviseccion. Acta Bioethica 2007; 13(1): 53-60.

15. Barrantes M, Mercado E. Aborto terapeutico en Nicaragua; el dialogo como parte de la solucion del conflicto. Acta Bioethica 2008; 14(1): 106-110.

16. Barrantes M, Rodriguez E, Lama A. Relacion medico paciente: derechos del adulto mayor. Acta Bioethica 2009; 15(2): 216-221. NIHMS\#160001.

17. Barrantes M. Genero, vejez y salud. Acta Bioethica 2006; 12(2): 193-197.

18. Borquez B. Consideraciones legales en la atencion del anciano. El consentimiento informado. Medwave October 2007; VII (9) electronic publication. Available at http://www.mednet.cl/link. cgi/Medwave/Enfermeria/Enfgeriatrica/8/2746

19. Bota A. Animales transgenicos como organismos artificiales. Acta Bioethica 2007; 13(1): 61-71.

20. Bota A. Contribucion de las normas CIOMS 2002 al desarrollo biotecnologico. La corresponsabilizacion del cientifico. Biological Research 2003; 36: 148-154. 
21. Bota A. El impacto de la biotecnologia en America Latina. Espacios de participacion social. Acta Bioethica 2003; 9(1): 21-38.

22. Bota A. El reto de la muestra biologica en los estudios farmacogeneticos. Acta Bioethica 2004; 10(2): 201-212.

23. Buendia AL, Alvarez de la Cadena-Sandoval C. Nivel de conocimiento de la bioetica en dos universidades de odontologia de America Latina. Acta Bioethica 2006; 12(1): 41-48.

24. Caceda-Gabancho K, Garcia CR. Aplicacion de consideraciones bioeticas en investigacion cientifica en estomatologia en pre y posgrado de la Universidad Peruana Cayetano Heredia en los ultimos 10 ańos. Revista Estomatologica Herediana 2011; 21(2): 73-78.

25. Cardozo CA. Hacia la definicion de una politica nacional de uso y cuidado de animales. Suma Psicologica 2010 Bogota; 17(1): 85-95.

26. Diaz E. Conocimiento de la Ley General de Salud respecto de las transfusiones sanguineas en medicos y pacientes Testigos de Jehova del Hospital Dr. Dario Contreras de Republica Dominicana. Acta Bioethica 2010; 16(1): 46-50. NIHMS\# 222375.

27. Estevez A. La cuestion del lego en etica de la investigacion. Revista Agora Philosophica 2006; 7(13): 45-66.

28. Estevez A. Principios de bioetica e investigacion. Ars Medica 2004; 9: 215-224.

29. Gamboa GA. Ecologia humana y Ecologia ambiental: binomio clave. Persona y Bioetica 2011; 15(1): 5-10.

30. Gamboa GA. La clave de la bioetica: la persona humana, su ser y su sentido. Revista de Bioetica Latinoamericana 2009; 2(1) http://www.saber.ula.ve/handle/123456789/26251

31. Gamboa GA. Relacion hombre y medio ambiente: un reto para la Bioetica. Revista Colombiana de Bioetica 2008; 3(1): 195-218.

32. Garbi-Novaes MR, Guilhem D, Lolas F. Ethical Conduct in Research Involving Human Beings In Brazil. Diagnosis of Research Ethics Committee. Arquivos de Medicina 2010; 23(4): 145-150. NIHMS222240.

33. Garbi-Novaes MRC, Garcez-Novaes LC, Guilhem D, Lolas F, Costa-Siveira CC, Vilar-Trindade EM, Shoiti-Komatsuri R, Guiottir MG. Ethical Attitudes of Brazilian Medical Students and Graduates with Active Methodologies. Revista Brasileira de Educacao Medica 2010; 34(1): 43-56. NIHMS\# 221987.

34. Garbi-Novaes MRC, Garcez-Novaes LC, Guilhem D, Lolas F, Silveira C, Giotti M. Analise da insercao dos temas de humanidades e etica, com metodologia de apredizagem baseada em problemas, em curriculo medico integrado em escola publica no distrito federal, Brasil. Acta Bioethica 2009; 15(2): 202-211. NIHMS\#160003.

35. Garbi-Novaes MRC, Guilhem D, Lolas F. Relato de 10 años de experiencia en comites de etica de investigacion de la Secretaria de Salud del Distrito Federal del Brasil. Acta Bioethica 2008; 14(2): 185-192. NIHMS\# 116000.

36. Garcia CR. Inclusion de la etica y la bioetica en la formacion de pre y posgrado del cirujanodentista en Peru. Acta Bioethica 2008; 14(1): 74-78. NIHMS\#115992.

37. Mondragon LB, Caballero MA. Del sujeto que ha intentado suicidarse y el Otro: la Institucion Psiquiatrica. Observaciones Filosoficas 2008; 7. Articulo electronico. http://www.observacionesfilosoficas.net/delsujetoquehaintentadosuiciBlueOneDVDripdarse.htm NIHMS\# 98207.

38. Mondragon LB, Caballero MA. La otredad, el intento de suicidio y la institucion psiquiatrica. Actas Españolas de Psiquiatria 2007; 34(1): 101-110.

39. Mondragon LB, Monroy Z, Ito ME, Medina-Mora ME. Disyuntivas en las concepciones sobre autonomia y beneficencia que afectan la terapeutica del intento suicida. Acta Bioethica 2010; 16(1): 77-86. NIHMS\# 203386

40. Mondragon LB, Romero M, Borges G. Etnography in an Emergency Room: Evaluating Patients with Alcohol Consumption. Salud Publica de Mexico 2008; 50(4): 308-315. 
41. Mondragon LB. Consentimiento informado: una praxis dialogica para la investigacion. Revista de Investigacion Clinica 2009; 61(1): 73-82. NIHMS\#158286.

42. Mondragon LB. Etica de la investigacion psicosocial. Revista de Salud Mental 2007; 30(6): 25-31.

43. Montoya G, Rodriguez E, Risco L, Valdebenito C, Schiattino I, Lolas F. Confidencialidad de la orientacion sexual en psiquiatria. Revista Chilena de Neuropsiquiatria 2006; 44(3): 170-177.

44. Montoya G. Bioetica y terapia electroconvulsiva. Revista de Psiquiatria Clinica 2005; 42(2): 1825.

45. Moreno L. Aspectos eticos de los estudios de biodisponibilidad y bioequivalencia de productos farmaceuticos contenidos en las legislaciones de America Latina. Acta Bioethica 2004; 10: $247-$ 260.

46. Ospina DE, Ivanovic F, Lolas F. Acompañando al paciente con trastorno bipolar: aportes a la psicoeducacion. Trastornos del Animo 2007; 3(1): 55-63.

47. Ospina DE, Ivanovic F, Lolas F. Trastorno bipolar desde la perspectiva de los familiares. Trastornos del Animo 2009; 5(1): 28-36. NIHMS\# 154145.

48. Rodriguez E, Valdebenito C, Misseroni A, Fernandez L, Outomuro D, Schiattino I, Lolas F. Percepciones sociales sobre genomica en cuatro paises latinoamericanos. Implicaciones etico legales. Derecho y Genoma 2004; 21: 141-164.

49. Rodriguez E, Valdebenito C, Misseroni A, Fernandez L, Outomuro D, Schiattino I, Ferrer M, Lolas F. Attitudes towards genomic research in four Latin American countries. Electronic Journal of Biotechnology 2005; 8(3). Available from: http://www.ejbiotechnology.info/content/vol8/issue3/ full/9/index.html ISSN 0717-3458.

50. Rodriguez K. Vision bioetica del VIH/SIDA en los Objetivos del Milenio 2010. Jurisprudencia Argentina, Numero Especial, 2011; 1.

51. Torres MA. Bioetica y ejercicio profesional de la odontologia. Acta Bioethica 2006; 12(1): 65-74.

52. Ulloa A, Barrantes M. Bioetica en Nicaragua. Acta Bioethica 2008; 14(2): 200-205. NIHMS\# 116005 .

53. Vidal MH. Cuestiones eticas y legales del uso de pruebas rapidas de diagnostico para VIH/SIDA domiciliarias. Revista Chilena de Salud Publica 2006; 10(2): 99-106.

54. Zwareva EC. Los comites de etica de investigacion biomedica y psicosocial en Bolivia. Acta Bioethica 2010; 16(1): 87-91. NIHMS\# 203380.

\section{Book, chapters}

1. Acuña-Johnson AP, Garbi-Novaes MRC. Formacion etica del farmaceutico. En: Garbi Novaes MR, Lolas F, Quezada A. Etica y Farmacia. Una perspectiva latinoamericana. Monografia de Acta Bioethica No 2. Santiago de Chile: CIEB Universidad de Chile, Programa de Bioetica OPS/OMS; 2009: 333-359.

2. Alvarez J, Cardozo CA. Etica de la investigacion biomedical que usa y cuida animales experimentales. En: Lolas F, Quezada A, Rodriguez E. (editores) Investigacion en Salud. Dimension Etica. Santiago de Chile: Santiago de Chile: CIEB Universidad de Chile; 2006: 239-248.

3. Alvarez J, Lolas F, Outomuro D. Historia de la etica en investigacion con seres humanos. En: Lolas F, Quezada A, Rodriguez E. (editores) Investigacion en Salud. Dimension Etica. Santiago de Chile: CIEB Universidad de Chile; 2006: 39-46.

4. Ayala-Jara CI. Justicia Sanitaria en el Peru. En: Leon FJ. (coordinador) Etica y Justicia Sanitaria en Situaciones de Catastrofe. Santiago de Chile: Sociedad Chilena de Bioetica; 2010: 65-70.

5. Barrantes M, Montoya G. Confidencialidad en salud e investigacion. En: Lolas F, Quezada A, Rodriguez E. (editores) Investigacion en Salud. Dimension Etica. Santiago de Chile: CIEB Universidad de Chile; 2006: 217-229. 
6. Barrantes M. Plasmaferesis: la sangre como mercancia. Book Chapter. Diccionario Latinoamericano de Bioetica. Bogota: UNESCO; 2008.

7. Bermudez de Caicedo C, Buendia AL, Deangelillo CA, Guerra AR. Los programas de formacion etica en odontologia. En: Cardozo C, Rodriguez E, Lolas F, Quezada A. (editores) Etica y Odontologia. Una Introduccion. Santiago de Chile: CIEB Universidad de Chile; 2007: 117-127.

8. Borquez B, Ciocca L, Lolas F, Romo F, Torres MA. Etica, bioetica, ley: conceptos basicos. En: Cardozo C, Rodriguez E, Lolas F, Quezada A. (editores) Etica y Odontologia. Una Introduccion. Santiago de Chile: CIEB Universidad de Chile; 2007: 19-30.

9. Borquez B, Jimenez P. Etica, derecho e investigacion. En: Lolas F, Quezada A, Rodriguez E. (editores) Investigacion en Salud. Dimension Etica. Santiago de Chile: CIEB Universidad de Chile; 2006: 47-58.

10. Borquez B, Triana J. Odontologia: profesion de riesgo. En: Cardozo C, Rodriguez E, Lolas F, Quezada A. (editores) Etica y Odontologia. Una Introduccion. Santiago de Chile: CIEB Universidad de Chile; 2007: 33-43.

11. Bota A, Estevez A, Fernandez L, Hernandez M, Hevia A, Lara CF, Moreno L, Rodriguez K. Comites de evaluacion etica y cientifica de la investigacion en seres humanos en los paises latinoamericanos y del Caribe. En: Lolas F, Quezada A, Rodriguez E. (editores) Investigacion en Salud. Dimension Etica. Santiago de Chile: CIEB Universidad de Chile; 2006: 59-80.

12. Campbell A, Alvarez JA. (editores) Bioetica en Perspectiva. Juarez, Mexico; Universidad Autonoma de Ciudad; 2008.

13. Cardozo CA, Mrad de Osorio A, Maldonado A, Cely G. (editores) Etica en investigacion. Una responsabilidad social. Colombia: Universidad Nacional de Colombia, Pontificia Universidad Javeriana; 2008.

14. Cardozo CA, Mrad de Osorio A, Martinez C, Rodriguez-Yunta E, Lolas Stepke F. El animal como sujeto experimental. Aspectos tecnicos y eticos. Santiago de Chile: Centro Interdisciplinario de Estudios en Bioetica (CIEB), Vicerrectoria de Investigacion y Desarrollo, Universidad de Chile; 2007.

15. Cardozo CA. El concepto salud-enfermedad oral: del ejercicio individual a la salud colectiva. Un dilema para el odontologo actual. En: Cardozo C, Rodriguez E, Lolas F, Quezada A. (editores) Etica y Odontologia. Una Introduccion. Santiago de Chile: CIEB Universidad de Chile; 2007: 45-52.

16. Cardozo CA. El dialogo y la comunicacion: una tarea urgente del cuerpo odontologico para el ejercicio colectivo. En: Cardozo C, Rodriguez E, Lolas F, Quezada A. (editores) Etica y Odontologia. Una Introduccion. Santiago de Chile: CIEB Universidad de Chile; 2007: 75-77.

17. Cardozo CA. Hacia la conformacion de una red de comites de etica de investigacion con animales en Latinoamerica. En: Leon FJ. (coord.) Etica Clinica y Comites de Etica en Latinoamerica. Santiago de Chile: Felaibe y Fundacion Ciencia y Vida; 2011: 43-52.

18. Cardozo CA. Los dilemas tecnicos, cientificos y eticos que enfrenta la odontologia actual. La funcion de los codigos de etica. En: Cardozo C, Rodriguez E, Lolas F, Quezada A. (editores) Etica y Odontologia. Una Introduccion. Santiago de Chile: CIEB Universidad de Chile; 2007: 99-104.

19. Castro-Romero JI. El seguro popular como politica publica de salud en Mexico. En: Leon FJ. (coordinador) Etica y Justicia Sanitaria en Situaciones de Catastrofe. Santiago de Chile: Sociedad Chilena de Bioetica; 2010: 55-60.

20. Cosmai NP. Confidencialidad y VIH. En: Leon FJ. (Coord.) Etica Clinica y Comites de Etica en Latinoamerica. Santiago de Chile: Felaibe y Fundacion Ciencia y Vida; 2011: 260-261.

21. Diaz E. Incidencia de Ley 42-01 en Transfusion Sanguinea a Testigos de Jehova: Beneficios de la Ley General de Salud en los Principios Fundamentales de la Bioetica. Editorial Academica Española; 2012.

22. Estevez A. Ambiguedad y cuestion moral. A proposito del ensayo de Viktor Von Weizsacker: Eutanasia y Experimentacion Humana". En: Lolas F. La Medicina Antropologica y el Juicio de Nurenberg. El aporte de Viktor Von Weizsacker. Monografia de Acta Bioethica No 5. Santiago de Chile: 
CIEB Universidad de Chile, Programa de Bioetica OPS/OMS; 2010: 179-188.

23. Fernandez-Manzano E, Garbi-Novaes MRC. La comunicacion y el dialogo: una tarea urgente de los farmaceuticos para fomentar la salud. En: Garbi Novaes MR, Lolas F, Quezada A. Etica y Farmacia. Una perspectiva latinoamericana. Monografia de Acta Bioethica No 2. Santiago de Chile: CIEB Universidad de Chile, Programa de Bioetica OPS/OMS; 2009: 313-332.

24. Gamboa G. Politicas recientes de poblacion. Book chapter. En: Velez Ramirez A. (editora) Poblacion, Vida y Desarrollo. Evidencia e Interrogantes en los albores del siglo XXI. Bogota: Universidad de La Sabana; 2008

25. Garbi-Novaes MRC, Lolas F, Quezada A. (organizadores) Etica e Farmacia. Uma abordagem latinoamericana em saude. Brasilia: Thesaurus Editores; 2009.

26. Garbi-Novaes MRC, Marcia-de-Oliveira H, Bernardino M, Rebem-de-Souza NN. Etica y calidad en la preparacion de medicamentos. En: Garbi Novaes MR, Lolas F, Quezada A. Etica y Farmacia. Una perspectiva latinoamericana. Monografia de Acta Bioethica No 2. Santiago de Chile: CIEB Universidad de Chile, Programa de Bioetica OPS/OMS; 2009: 105-126.

27. Garbi-Novaes MRC, Reyes-Matheus CM, Delfino-de-Faria AV. Etica en la farmacoterapia y corrientes de la medicina contemporanea. En: Garbi Novaes MR, Lolas F, Quezada A. Etica y Farmacia. Una perspectiva latinoamericana. Monografia de Acta Bioethica No 2. Santiago de Chile: CIEB Universidad de Chile, Programa de Bioetica OPS/OMS; 2009: 73-104.

28. Garbi-Novaes MRC. O Comite de Etica em Pesquisa. In: Guilhem D, Sorokin P, Queiroz W, Zicker F, (editors) Etica em Pesquisa: compartilhando responsabilidades. Brasilia: Letras Livres, Editora UnB; 2008.

29. Gilhem D, Garbi-Novaes MRC. Etica e investigacion con seres humanos. En: Garbi Novaes MR, Lolas F, Quezada A. Etica y Farmacia. Una perspectiva latinoamericana. Monografia de Acta Bioethica No 2. Santiago de Chile: CIEB Universidad de Chile, Programa de Bioetica OPS/OMS; 2009: 173-198.

30. Irribarra R, Rodriguez E, Torres MA. Bioetica, bioseguridad y responsabilidad social: nuevo campo de reflexion para el odontologo En: Cardozo C, Rodriguez E, Lolas F, Quezada A. (editores) Etica y Odontologia. Una Introduccion. Santiago de Chile: CIEB Universidad de Chile; 2007: 129-138.

31. Jimenez-Sanchez G, Lara C, Arellano-Mendez A. A survey of the development of mexican bioethics: genomic medicine as one of its greatest challenges. En: Pessini L, De Barchifontaine C, Lolas Stepke F. (editors). Iberoamerican Bioethics. History and Perspectives. Heidelberg: Springer Dordrecht; 2010: 159-174.

32. Jimenez-Sanchez G, Lara C. La medicina genomica y el derecho a la proteccion de la salud. Book Chapter. Derecho y medicina. Intersecciones y convergencias en los albores del siglo XXI. $1^{\text {a }} \mathrm{ed}$. Mexico: Suprema Corte de Justicia de la Nacion; 2009; 471-491.

33. Lara C. La necesidad de regulacion en paises como Mexico. Book Chapter. Comites de etica de investigacion biomedica en paises en desarrollo. Mexico: Instituto Nacional de Salud Publica; 2007.

34. Lara CF. Normas legales para los comites de etica de la investigacion cientifica. En: Lolas F, Quezada A, Rodriguez E. (editores) Investigacion en Salud. Dimension Etica. Santiago de Chile: CIEB Universidad de Chile; 2006: 81-91.

35. Lescano AR, Moreno L, Zavala S, Luza A, Litewka S. Capacitacion en etica de la investigacion. Tendiendo puentes entre la industria, los investigadores y los comites. En: Leon FJ. (coord.) Etica Clinica y Comites de Etica en Latinoamerica. Santiago de Chile: Felaibe y Fundacion Ciencia y Vida; 2011: 86-91.

36. Mondragon L, Rodriguez E, Valdebenito C. Etica de la investigacion en ciencias sociales. En: Lolas F, Quezada A, Rodriguez E. (editores) Investigacion en Salud. Dimension Etica. Santiago de Chile: CIEB Universidad de Chile; 2006: 93-101.

37. Mondragon LB, Ito ME, Monroy Z, Netza C, Ocampo R, Rodriguez E, Larios N, Lolas F, Lara 
CF, Borquez B. Guia bioetica para el tratamiento clinico de pacientes que han intentado suicidarse. Mexico: Instituto Nacional de Psiquiatria Ramon de la Fuente Muñiz, Secretaria de Salud; 2010. ISBN 978-607-460-116-9.

38. Mondragon LB. El lugar imprescindible de la bioetica en la practica de saberes cientificos. Cap. 7. Book Chapter. Monroy Nasr Z, Leon Sanchez R. Epistemologia, Psicologia y Enseñanza de la Ciencia. Mexico: Facultad de Psicologia, UNAM; 2009.

39. Montoya G. Poblaciones especiales en investigacion biomedica. En: Lolas F, Quezada A, Rodriguez E. (editores) Investigacion en Salud. Dimension Etica. Santiago de Chile: CIEB Universidad de Chile; 2006: 191-214

40. Moreno L, Fuentes D, Rodriguez J. Reglamento de ensayos clinicos en Peru y prioridades de investigacion. En: Lolas F. (editor) Dimensiones eticas de las regulaciones en salud. Monografia de Acta Bioethica No 3. Santiago de Chile: CIEB Universidad de Chile, Programa de Bioetica OPS/ OMS; 2009: 325-352.

41. Nieto V. Curso de formacion en bioetica para funcionarios de la gerencia de salud del IPS, Montevideo. En: Leon FJ. (editor) Docencia de la Bioetica en Latinoamerica. Experiencias y valores compartidos. Santiago de Chile: Felaibe y Fundacion Ciencia y Vida; 2011: 366-379.

42. Nieto V. La Reforma de la Salud en La Republica Oriental del Uruguay. En: Leon FJ. (coordinador) Etica y Justicia Sanitaria en Situaciones de Catastrofe. Santiago de Chile: Sociedad Chilena de Bioetica; 2010: 50-54.

43. Rodriguez E, Moreno L. Los principios eticos y la conduccion responsable de la investigacion. En: Lolas F, Quezada A, Rodriguez E. (editores) Investigacion en Salud. Dimension Etica. Santiago de Chile: CIEB Universidad de Chile; 2006: 279-292.

44. Rodriguez K. Historical aspects of the development of bioethics in Ecuador. Book Chapter. Pessini L, De Barchifontaine C, Lolas-Stepke F. (editors) Iberoamerican Bioethic. History and Perspectives. Heidelberg: Springer Dordrecht; 2010: 149-158.

45. Rodriguez-Paz ML. Sistema de Salud en Colombia. En: Leon FJ. (coordinador). Etica y Justicia Sanitaria en Situaciones de Catastrofe. Santiago de Chile: Sociedad Chilena de Bioetica; 2010: 6164.

46. Sanders M. Reflexiones acerca de la enseñanza de la bioetica en Argentina. En: Leon FJ. (editor) Docencia de la Bioetica en Latinoamerica. Experiencias y valores compartidos. Santiago de Chile: Felaibe y Fundacion Ciencia y Vida; 2011: 110-121.

47. Torres MA. Bioetica e investigacion en odontologia. En: Cardozo C, Rodriguez E, Lolas F, Quezada A. (editores) Etica y Odontologia. Una Introduccion. Santiago de Chile: CIEB Universidad de Chile; 2007: 79-87.

48. Torres MA. Bioetica e investigacion en odontologia. En: Lolas F, Quezada A, Rodriguez E. (editores) Investigacion en Salud. Dimension Etica. Santiago de Chile: CIEB University of Chile; 2006: 249-256.

- In preparation: Etica de la Investigacion Biomedica y Psicosocial, Lolas F, Rodriguez E, Quezada A. (editores). Centro Interdisciplinario de Estudios en Bioetica (CIEB), Universidad de Chile, 2011.

- (48) Castro-Romero JI.: Bioetica e investigacion en cancer.

- (49) Garbi-Novaes MRC.: Etica de los ensayos clinicos multicentricos.

- (50) Nieto V.: El menor como sujeto de investigacion

- (51) Ospina-Muñoz DL. Rodriguez-Yunta E.: Reflexion sobre etica de la investigacion psicosocial.

- (52) Vidal-Anzardo MH.: Dilemas eticos actuales en la investigacion en SIDA. 


\section{In the web}

1. Ayala-Jara C. Reglamento del Comite Institucional de Etica de en Investigacion de la Universidad Nacional de Trujillo, Peru (2011). Disponible en http://www.bioetica.uchile.cl/doc/reglamento_ ciei_unt.pdf

2. Bota A, Estevez A, Fernandez L, Hernandez M, Hevia A, Lara C, Moreno L, Rodriguez K. Los Comites de Evaluacion Etica y Cientifica de la Investigacion en Seres Humanos en los Paises Latinoamericanos y el Caribe: Una Propuesta para su Desarrollo (2004). Disponible en http://www.bioetica. uchile.cl/cursos/docs.htm

3. Cardozo CA, Mrad de Osorio A. Derechos Humanos, Derechos de la Mujer: Ante todo el Principio de Proteccion. Ministerio de Proteccion Social 2007; Convenio 405; www.unal.edu.co/bioeti$\mathrm{ca} /$ documentos/conveniodoc/a1_presentaciona.doc

4. Cardozo CA. Declaracion OPS sobre requerimientos exigibles a los autores de investigaciones en las cuales se involucre el uso y cuidado de animales experimentales (2005). Disponible en http://www. bioetica.uchile.cl/cursos/docs.htm

5. Espinoza-Perez VE, Rodriguez-Yunta E, Sanchez AL. Situacion de los Comites de Etica de la Investigacion en Salud en Honduras (Lima, 2011). Disponible en http://www.bioetica.uchile.cl/doc/ Comites_Etica_Honduras.pdf

6. Ethics of Biomedical and Psicosocial Research Program, Interdisciplinary Center for Studies on Bioehics, University of Chile, Grant Fogarty International Center R25TWOO6056, Report of an Evaluation Meeting (Lima, 2011). Disponible en http://www.bioetica.uchile.cl/doc/Report_Lima_Meeting. pdf

7. Fernandez L. Guias operacionales para el funcionamiento de un Comite Etico Cientifico (2004). Disponible en http://www.bioetica.uchile.cl/cursos/docs.htm

8. Gamboa-Bernal GA. Regulacion de la etica de investigacion en Colombia (Lima, 2011). Disponible en http://www.bioetica.uchile.cl/doc/Regulacion_Colombia.pdf

9. Garbi-Novaes MRC, Pastor-Martinez E, Cañete R. Relatos de reacciones adversas a medicamentos en ensayos clinicos experimentales con nuevos farmacos en Brasil (Lima, 2011). Disponible en http:// www.bioetica.uchile.cl/doc/Relatos_reacciones_adversas.pdf

10. Informe de Reunion de Evaluacion de Egresados de Programa de Etica de la Investigacion del Centro Interdisciplinario de Estudios en Bioetica de la Universidad de Chile, Grant Fogarty International Center R25TWOO6056 (Lima 2011). Disponible en http://www.bioetica.uchile.cl/doc/Informe_reunion_Lima.pdf

11. Lara CF. Estudio de Caso: Evaluacion piloto de dos (2) regimenes antirretrovirales en un entorno de escasos recursos (2004). Instituto Tecnologico de Santo Domingo, Republica Dominicana. Disponible en http://www.bioetica.uchile.cl/cursos/docs.htm

12. Lara CF. Propuesta de Proyecto Norma Oficial Mexicana en Materia de Comisiones de Etica para su Fortalecimiento (2004). Disponible en http://www.bioetica.uchile.cl/cursos/docs.htm

13. Moreno L. Estudio de Caso: Terapia de irradiacion sanguinea con luz ultravioleta en personas viviendo con VIH/ SIDA en Republica Dominicana (2004). Instituto Tecnologico de Santo Domingo, Republica Dominicana. Disponible en http://www.bioetica.uchile.cl/cursos/docs.htm

14. Moreno L. Reglamento del Comite de Etica del Instituto Dermatologico y Cirugia de Piel Dr. Huberto Bogaert Diaz (2004). Disponible en http://www.bioetica.uchile.cl/cursos/docs.htm

15. Moreno L. Reglamento interno del Comite de Etica en materia de investigacion del Hospital General Plaza de la Salud de Santo Domingo (2004). Disponible en http://www.bioetica.uchile.cl/cursos/ docs.htm

16. Ospina D, Ivanovic F, Lolas F. Acompañando el trastorno bipolar. Aportes a la psicoeducacion. $8^{\circ}$ Congreso Virtual de Psiquiatria, INTERPSIQUIS 2007. http://www.psiquiatria.com

17. Rodriguez E, Lolas F, Castro JI, Garbi-Novaes MR, Gamboa G, Moncayo LP, Diaz E, Valencia- 
Marroquin HE, Rodriguez K. Comisiones Nacionales de Bioetica y Organismos Nacionales de Desarrollo de Ciencia y Tecnologia. Una Reflexion. Lima (2011). Disponible en http://www.bioetica. uchile.cl/doc/Comisiones_nacionales_Bioetica.pdf

18. Rodriguez E, Lolas F, Garbi-Novaes MR, Cardozo CA, Castro JI, Rodriguez K, Diaz E, Moncayo LP, Mondragon L, Valencia-Marroquin HE. Integridad Etica en la Investigacion en Latinoamerica (Lima, 2011). Disponible en http://www.bioetica.uchile.cl/doc/Integridad_investigacion.pdf

19. Rodriguez K. Linea de base de funcionalidad de comites de etica de la investigacion en Quito, Ecuador (2004). Disponible en http://www.bioetica.uchile.cl/cursos/docs.htm

20. Sanders MM. El estado de la enseñanza de la bioetica en Argentina (2012). Disponible en http:// www.eps-salud.com.ar/bioetica-argentina.htm

21. Translation in to Spanish of Manual: Research ethics committees Basic concepts for capacity-building. World Health Organization (2009). Disponible en http://www.bioetica.uchile.cl/doc/comite_etica_investigacion_who.pdf

22. Translation Informed Consent Documents WHO (2008). Disponible en http://www.bioetica.uchile. cl/doc/Consentimiento-Clinical.pdf; http://www.bioetica.uchile.cl/doc/Consentimiento-Informado-almacenamiento-muestras.pdf; http://www.bioetica.uchile.cl/doc/Consentimiento-Asentimiento.pdf; http:// www.bioetica.uchile.cl/doc/Consentimiento-Informado-Parental.pdf

23. Vidal MH. Aprobacion de test caseros para VIH. Critica Etica: Pautas de Emanuel (2005). http:// www.bioetica.uchile.cl/cursos/docs.htm

\section{Online virtual modules on ethics of research (CITI Program)}

1. Bota A, Estevez A, Fernandez L, Hernandez M, Hevia A, Lara C, Moreno L, Rodriguez K. Comites de evaluacion etica y cientifica de la investigacion con seres humanos en Latinoamerica y su normativa. Available at http://www.citiprogram.org

2. Cardozo CA. Comites de etica de investigacion en Colombia. Available at http://www.citiprogram. org

3. Cardozo CA. Etica de la investigacion biomedica en animales experimentales. Available at http:// www.citiprogram.org

4. Montoya G. Investigacion con seres humanos. Poblaciones especiales en Latinoamerica. Available at http://www.citiprogram.org

5. Ospina D. Etica en la investigacion psicosocial. Available at http://www.citiprogram.org

6. Rodriguez E. Translation and adaptation for Latin America of Responsible conduct of research and ethics of biomedical research modules of citiprogram University of Miami, U.S.

\section{Web bulletins}

1. Borquez-Polloni B. Bulletin Legal Reports. National Library of Congress, Chile. Available at http:// www.bcn.cl/pags/publicaciones/serie

2. Buendia AL, Cardozo CA. Bulletin Reactivos Biologicos, Asociacion Chilena para la Ciencia de Animales de Laboratorio, octubre 2005.

3. Cardozo CA. Bulletin Bioetica y Salud Oral, November 2005. Available at http://www.bioetica. ops-oms.org/E/ehome.htm 


\section{Web sites}

1. Cardozo CA. Bioethics net National University Colombia. Available at http://www.unal.edu.co/ bioetica

2. Rodriguez H. Creation of a website for bioethics at Department of Medicine Republic University, Montevideo. Available at http://www.bioetica.edu.uy

3. Torres MA. Scientific Ethical Review Committee. Available at http://odontologia.uchile.cl/? $\mathrm{nfpb}=$ true\&_pageLabel=conUrlOdontologia\&url=51001

\section{Additional research projects presented}

1. Barrantes M, Bertiroti A, Borquez B, Cardozo CA, Eckel W, Feinholz D, Lolas F, Mancini R, Mondragon LB, Mordini E, Ormaeche M, Rodriguez E, Villaveces JL, Vidal-Anzardo M. Ethics development in biomedical and psychosocial research in five Latin American countries in relation to European Union: One step further towards international competition. Sixth Frame Program European Market Activity, SiS 2005, Coordination Actions Capacity building on ethics in developing countries.

2. Barrantes M, Mercado E. National formation of a Scientific Ethical Review Committee. MINSA, Nicaragua (2007-2008).

3. Barrantes MC, Romeo Casabona C, Eckel W, Mercado E, Cardozo CA, Mondragon LB, Mordini E, Lara CF, Lolas F, Moreno L, Rodriguez E. Role of ethical review on quality, social legitimacy and applicability of scientific research at Latin America. Learning from the European experience. Seventh Frame Program European Market Activity 5.1.2 Broader engagement to anticipate and clarify political, societal and ethical issues, SiS 2007 1.2.2.4 Capacity building on ethics in developing countries.

4. Bota A. EULABOR Latin American and European Ethical Regulation Systems of Biomedical Research: Comparative Analysis of their Pertinence and Application for Human Subjects Protection. Frame Program 6 of the European Union (2005-2007).

5. Buendia AL. Knowledge of ethics and bioethics in Schools of Dentistry, U.S. and Mexico. University of Southern California, School of Dentistry (2007).

6. Cardozo CA. Discussion Forum on Sentence C355 about Abortion in Colombia under a Medical, Legal and Ethical Perspective. National University of Colombia (2007-2008).

7. Cardozo CA. Integration of basic and ethical neuroscientific aspects in neuropsiquiatry health care. Colombian Macro Universities Web (2007-2008).

8. Cardozo CA. Maltreatment and intrafamiliar violence: looking for a public policy from a bioethics perspective. UNPFA, Bogota, Colombia (2008-2010).

9. Gamboa G. Quality of life in clinical practice. University Clinic Teleton, Chia; Hospital Tunal, Bogota; Hospital Pablo Tobon Uribe, Medellin; Clinica Versalles, Cali; Medical Center Imbanaco, Cali. University La Sabana, Bogota, Colombia (2008-2009).

10. Garcia CR. Application of ethical issues in oral health scientific research last ten years at Cayetano Heredia Peruvian University. Lima, Peru (2008-2009).

11. Garcia CR. Evaluation of informed consent process applied to patients in Oral Health Clinic at Cayetano Heredia Peruvian University. Lima, Peru (2008-2009).

12. Lolas F, Rodriguez E, Misseroni A, Outomuro D, Schiattino I, Valdebenito C, Fernandez L, Montoya G. Grant Department of Energy award DE-FG02-02R 63435. Representation of Genomics Research among Latin American Laymen and Bioethics: A inquiry into the migration of knowledge and its impact on underdeveloped communities. Office of Environmental and Biological Research. Human Genome Program. Department Of Energy, United States (2002-2005). 
13. Mondragon L. Bioethics and Mental Health: Bioethical Dilemmas in the Therapeutics of Suicidal Attempt, 4601 N. National Psychiatric Institute Ramon de la Fuente Muñiz (Mexico, 2007-2008).

14. Ormaeche M. Collective Informed Consent in Indigenous Populations. Dept. of Epidemiology, Ministry of Health, Lima, Peru (2007-2009).

\section{Health research policies and regulations developed}

1. Ayala C. Creation scientific ethical review committee at Trujillo National University. Trujillo, Peru. Presidential Resolution N 1326-2010/UNT. 16 members, October 2010.

2. Ayala C. Proceeding Norms Institutional Scientific Ethical Review Committee Trujillo National University. Trujillo, Peru (2010).

3. Barrantes M. Proposal Norm for reviewing research protocols at Nicaragua. National Health Institute, Nicaragua (2008).

4. Cardozo CA. Agreement 405/2006: Legal, ethical and medical implications for sentence C355 on abortion not penalized exceptional cases (2006).

5. Cardozo CA. Ethics and Good Management Code UniSalud. Colombia National University (February 2010).

6. Cardozo CA. PAHO Declaration on requirements for investigators in research involving the use and care of experimental animals (2005). Available at http://www.bioetica.uchile.cl/doc/animales.pdf

7. Diaz S. Conformation Scientific Ethical Review Committee Universidad Alberto Masferrer, El Salvador. (November 2010).

8. Diaz S. Proposal National Rules for regulating research involving human beings at El Salvador (2007).

9. Fernandez L. Operational guidelines for scientific ethical review committees functioning. Santiago, Chile (2004). Available at http://www.bioetica.uchile.cl/cursos/docs.htm

10. Gamboa GA. Concept Project Law Creation of Observatory for reproductive and sexual rights (2011).

11. Gamboa GA. Norms for scientific ethical review committee of Faculty of Medicine, Universidad de la Sabana, Bogota, Colombia (2009).

12. Lara CF. Project Official Mexican Norm for Ethical Review Commissions (2004). Available at http:// www.bioetica.uchile.cl/cursos/docs.htm

13. Moncayo LP. Accreditation Requirements and Procedures for Scientific Ethical Review Committees in Ecuador. Public Health Ministry Science and Technology, Bioethics component (2010).

14. Moncayo LP. Bioethical Norms for research involving human beings in Ecuador. Public Health Ministry Science and Technology, Bioethics component (2010).

15. Moncayo LP. Norms of Procedure for Scientific Ethical Review Committee. Public Health Ministry Science and Technology, Bioethics component, Ecuador (2011).

16. Mondragon L, Lara CF, Borquez B. Ethical considerations for suicidal prevention. Document Suicidal Prevention Program. National Institute of Psychiatry, Health Secretary, Mexico (2009).

17. Moreno L. Norms for scientific ethical review committee of Dermatology and Skin Surgery Institute Dr. Huberto Bogaert Diaz, Santo Domingo, Dominican Republic (2004). Available at http://www. bioetica.uchile.cl/cursos/docs.htm

18. Moreno L. Norms for scientific ethical review committee of General Plaza Hospital, Santo Domingo, Dominican Republic (2004). Available at http://www.uchile.cl/bioetica/doc/reglamento. pdf

19. Ormaeche M. Second Generation Epidemiological Surveillance STD, HIVIAIDS and Hepatitis B in pregnant and partner Indigenous Amazon Population. Direccion General de Epidemiologia, Ministerio de Salud, Peru (2009).

20. Rodriguez K. Operational Guidelines Scientific ethical review committee Pontifitial Catholic University, Quito, Ecuador (2005). 
21. Sanabria-Ortega CW. Guidelines for National Bioethics Committee in Paraguay (2009).

22. Torres MA. Norms for scientific ethical review committee of Dentistry Dept. University of Chile (2008). Available at http://odontologia.uchile.cl/?_nfpb=true\&_pageLabel=conUrlOdontologia\&u $\mathrm{rl}=51001$

23. Ulloa A. Norms for scientific ethical review committee of National Autonomous University of Managua (UNAM), Managua, Nicaragua (2008).

24. Ulloa A. Project Official Nicaraguan Norm for Ethical Review Committees (2009)

25. Valencia HA. Standard Procedures Scientific Ethical Review Committee University of San Carlos, Guatemala (2010).

26. Vidal MH. Proposal Technical Norm Use of Biological Samples in Clinical Trials National Institute of Health, Peru (2011): Number INS/OGAT-V.01.

\section{Workshops and interventions (developed by trainees and supported by CIEB Fogarty Program)}

1. Workshop: Bioethics for staff Social Security Institute, Montevideo, Uruguay, October 2010 (trainee Nieto V., advisor: L. Rueda).

2. Workshop: Current ethical topics Health Research, University El Valle, Cali, Colombia, November 23-25, 2009 (trainee Rodriguez ML., advisor: E. Rodriguez, K. Lagos).

3. Workshop: Ethical issues in genetic research University La Republica, Montevideo, Uruguay, June 2010 (trainee: Rodriguez H., advisor: E. Rodriguez).

4. Workshop: Ethics in Reproductive Health, National University of Colombia, October 2011 (trainees: Maldonado O., Cardozo CA.; advisor : K. Lagos).

5. Workshop: Ethics in scientific research for scientific ethical review committee National University of Trujillo, Peru September, 2011 (trainee: Ayala C., advisor: E. Rodriguez).

6. Workshop: Ethics of Research and Health Care for the Elderly, Fundacion Santa Fe, Bogota, Colombia, October 2010 (trainee Cardozo CA., advisor: F. Lolas).

7. Workshop: Ethics of research capacitating scientific ethical review committees, Hospital Dario Contreras, CONYCIT Dominican Republic, Santo Domingo, April, 2010 (trainee Diaz E., advisor: E. Rodriguez).

8. Workshop: Ethics of Research for Scientific Ethical Review Committees Faculty of Medicine University of Antioquia, Medellin, Colombia, June 2011 (trainees: Montoya G., Ospina D., advisor: E. Rodriguez).

9. Workshop: Ethics of research with human subjects address to pediatricians involved in research Hospital Notti, Mendoza, Argentina, in collaboration with National University of Cuyo, June 2010 (trainees: Melonari PA. \& Pavon S., advisor: E. Rodriguez).

10. Workshop: Human Rights, Bioethics and Handicaps. Social Security Institute, Montevideo, Uruguay, November 2010 (trainee: Nieto V., advisor: L. Rueda).

11. Workshop: Human subjects research protection and responsible research conduct, Tegucigalpa, Honduras, 24-25 June, 2010 (trainee Merlo AR., advisor: F. Lolas).

12. Workshop: Informed consent and scientific ethical review committees, Hospital Simon Bolivar, Bogota, Colombia, October 2009 (trainee: Gamboa G., advisor: E. Rodriguez).

13. Workshop: Organization and implementation of ethics of research for the formation of scientific ethical review members in order to establish a scientific ethical review committee at Trujillo National University, Trujillo, Peru, October 2010 (trainee: Ayala CI., advisor: E. Rodriguez, K. Lagos). Agreement for cooperation on research ethics between Trujillo National University, Trujillo, Peru, and Interdisciplinary Center for Studies on Bioethics, University of Chile, 2010-2011.

14. Workshop: Protection of Research Human Subjects according to Norms and Regulations at Quito and Guayaquil, Ecuador, October 12-15, 2009 (trainee Moncayo LP., advisor: E. Rodriguez). 
15. Workshop: Scientific and Hospital Ethical Review Committees, Health Research Regulations, Professional ethics codes, Ministry of Health, National University San Carlos, OPS Guatemala, April, 2010. (trainee Valencia HA., advisor: E. Rodriguez, K. Lagos).

16. Workshop: Seminar ethics of research and scientific ethical review committees, Pontifitial Catholic University, Quito Ecuador, October 2005 (trainee: Rodriguez K., advisor: R. Mancini).

17. Workshop: Seminar Ethics of Research Regulations \& Scientific Integrity in Latin America for former trainees, September 2011, National Institute of Health, Lima, Peru: Evaluation Meeting of the CIEB Fogarty Program.

18. Workshop: Seminar Ethics of Social Research, School of Transborder Studies, Arizona State University, November 2011 (trainee: Garcia-Perez MH.).

19. Workshop: Ethics of research, Health Care Services, Ministry of Health, April 2012 (trainee Marimon-Diaz Y.).

20. Workshop: How to present a project to the scientific ethical review committee, for researchers Dentistry Department University of Chile, April 2012 (trainee: Torres, M.A., advisor: E. Rodriguez).

- Trainees:

- Ayala-Jara Carmen I.: Progress ethics of research at National University of Trujillo, Peru.

- Cardozo, Carmen A.: Activities carried out in ethics of research National University of Colombia.

- Castro-Romero, Julieta I.: Strengthening and experience of scientific ethical review committee of Public

Health National Institute, Mexico.

- Diaz-Santana, E.: Analysis of the development of ethics of research regulations Dominican Republic.

- Espinoza P., Vilma E.: Scientific ethics of health research committees situation at Honduras.

- Gamboa-Bernal, Gilberto A.: Ethics of research regulations at Colombia.

- Garcia-Rupaya, Carmen R.: Ethics of research curricula development Dentistry Department Cayetano Heredia Peruvian University, Peru.

- Garbi-Novaes, Maria R.: Development Scientific Ethical Review Committee Health Secretary Federal District, Brazil.

- Lara-Alvarez, Cesar F.: Ethics of Biomedical and Psychosocial Research.

- Lara-Alvarez, Cesar F.: Scientific Ethics of Research Committees Functioning and Regulation Guidelines.

- Moncayo, Lilian P.: Development process of science and technology, bioethics component, Public Health Ministry, Ecuador.

- Mondragon-Barrios, Liliana: Development ethics of research Program National Institute of Psychiatry Ramon de la Fuente Muñiz, Mexico.

- Moreno, L.: Ethics of research regulations at Peru.

- Ormaeche-Macassi, Melvy: Rights to freedom of determination towards development, participation, consulting and consent in the process of building rights to guarantee indigenous populations health.

- Ospina-Muñoz, Doris E.: Bioethics and Biolaw.

- Pavon, Sandra: Vulnerable populations in mental health.

- Rodriguez S., Katya: Ethics of research development at Ecuador.

- Valencia-Marroquin, Hilda E.: Ethics of research experience at San Carlos University, Guatemala.

- Advisors:

- Lescano, Roxana: Scientific Ethics of Research Committees Functioning and Regulation Guidelines.

- Litewka, Sergio: Pan American Bioethics Initiative. Collaborating in the development of ethics of research training programs.

- Lolas, Fernando: Ethics of research future developments.

- Lolas, Fernando: Institutionalization scientific research.

- Rodriguez, Eduardo: Ethics of Biomedical and Psicosocial Research Program at CIEB, University of Chile, Developmet 2002-2011, Fogarty Grant. 
21. Workshop: Ethics of Research and Responsibility, Buenos Aires, November 2011 (sponsor by University Austral, CIEB and Fogarty International Center, trainees: Cosmai N., Sanders-Bruletti MM., advisor: F. Lolas).

22. Workshop: First International Meeting on Bioethics and Research for Legislators, Buenos Aires, Argentina, November 201 (sponsor by CIEB, University of Flores and Fogarty International Center, trainee: Sanders-Bruletti MM., advisor: E. Rodriguez).

\section{Training programs on research ethics developed by trainees within the framework of the CIEB Fogarty Grant}

1. Ayala-Jara C.: Ethics of Research course at National University of Trujillo, Peru, 2011-.

2. Barrantes-Monge M.: Ethics of research curricula Medicine Department National Autonomous University Nicaragua, 2009-2010.

3. Cardozo CA.: Ethics of research program National University of Colombia, Bogota, Colombia, 2009-.

4. Cardozo CA.: Permanent Seminar Ethics of Research National University of Colombia, Bogota, Colombia, 2007-.

5. Gamboa G.: Course Good Clinical Practice and Scientific Ethical Review Committees University La Sabana, Bogota Colombia, 2010-.

6. Gamboa G.: Modules bioethics Clinical Pharmacology and Medicine Department University La Sabana, Bogota Colombia, 2009-.

7. Garbi-Novaes MR.: Courses ethics of research Interdisciplinary Postdegree Program University of Brazilia, 2009-2010.

8. Garbi-Novaes MR.: Ethics of research Program Interdisciplinary Postdegree Program University of Brazilia, 2010-

9. Garcia-Rupaya C.: Coordination short course ethics of research for dentistry students at Cayetano Heredia Peruvian University, Lima Peru 2007-2008.

10. Garcia-Rupaya C.: Training course ethics of research curricula dentistry Department Cayetano Heredia Peruvian University, Lima Peru, 2008-.

11. Mercado-Morales E.: Ethics of research curricula Medicine Department National Autonomous University Nicaragua, 2009-.

12. Mondragon L.: Courses and seminars ethics of research National Institute of Psychiatry Ramon de la Fuente Muñiz, Mexico, 2008-.

13. Mondragon L.: Development of a seminar for academics on ethics of psychosocial research, Psychiatric Institute Ramon de la Fuente Muñiz, Mexico, 2010-.

14. Mondragon L.: Ethics of research program National Institute of Psiquiatry Mexico, 2010-.

15. Nieto V.: Bioethics curricula for Medicine Department, University La Republica, Montevideo, Uruguay, 2011-.

16. Ospina D.: Program BioLaw Research: Law Department University Santo Tomas, Medellin, Colombia, 2011-.

17. Sanders-Bruletti MM.: Bioethics Program Law Department University Flores, Buenos Aires, Argentina, 2011-.

18. Torres-Quintana MA.: Courses for Bioethics training in Dentistry School, University of Chile, 2008-.

19. Valencia-Marroquin de Abril H.: Development curricula ethics of research courses San Carlos University, Guatemala, 2011-.

20. Vidal-Anzardo M.: Ethics of research course in Research Program ADIECS San Marcos Mayor National University, Peru 2011-. 


\section{Creation of new scientific ethical review committees by interventions of trainees}

1. Ayala-Jara C.: Scientific Ethical Review Committee National University of Trujillo, Peru, 2010-.

2. Diaz S.: National Bioethics Commission El Salvador, 2008.

3. Diaz S.: Scientific Ethical Review Committee University Alberto Masferrer, El Salvador, 2009.

4. Melonari P.: Scientific ethics of research Committee Sociedad Argentina Filial Mendoza, Argentina, 2009.

5. Merlo A. Scientific ethical review committee National Autonomous University of Honduras, Valle de Sula, 2012.

6. Moreno-Exebio L.: Scientific ethical review committee of Dermatology and Skin Surgery Institute Dr. Huberto Bogaert Diaz, Santo Domingo, Dominican Republic, 2004.

7. Moreno-Exebio L.: Scientific ethical review committee of General Plaza Hospital, Santo Domingo, Dominican Republic, 2004. Available at http://www.uchile.cl/bioetica/doc/reglamento.pdf

8. Rodriguez-Sanchez K.: Scientific Ethical Review Committee Pontifitial Catholic University, Quito, Ecuador, 2005.

9. Torres MA.: Scientific Ethical Review Committee Dentistry Department University of Chile, 2008.

\section{Participation of trainees in scientific ethical review committees}

1. Ayala-Jara C.: President Scientific Ethical Review Committee National University of Trujillo, Peru, 2010-.

2. Barrantes-Monge M.: Member Scientific Ethical Review Committee National Autonomous University, Nicaragua, 2008-.

3. Beltran-Sarmiento C.: Member Scientific Ethical Review Committee University La Sabana, Bogota, Colombia, 2009-.

4. Borquez-Polloni B.: Member Scientific Ethical Review Committee Ministry of Health West Service, Santiago, Chile, 2007-.

5. Buendia AL.: Member Scientific Ethical Review Committee School of Dentistry University of Southern California, 2008-.

6. Cardozo CA.: Member Scientific Ethical Review Committee National University of Colombia, 2006-; Consultant Scientific ethical review committees: University Clinic Valle de Lili, Hospital Federico Lleras Acosta, Hospital Fusagasuga, University Foundation Area Andina, Dermatology Hospital Bogota, 2008-.

7. Diaz S.: Member Scientific Ethical Review Committee Public Health Major Council, El Salvador, 2007-.

8. Diaz-Santana E.: Member Scientific Ethical Review Committee Infectious Diseases Foundation, Dominican Republic, 2009-; Vicepresident Scientific Ethical Review Committee Center for Diagnosis and Advanced Medicine and Telemedicine (CEDIMAT), Dominican Republic, 2009-.

9. Estevez A.: Member Scientific Ethical Review Committee Universidad Nacional del Sur, Argentina, 2005-.

10. Fernandez-Milla L.: President Scientific Ethical Review Committee Ministry of Health Servicio Salud Metropolitano Sur, Santniago, Chile, 2004-2009.

11. Gamboa-Bernal G.: President Scientific Ethical Review Committee Universidad La Sabana, Colombia, 2007-.

12. Garbi-Novaes MR.: Member Scientific Ethical Review Committee Secretaria de la Salud Distrito Federal, Brazil.

13. Garcia-Rupaya CR.: Member Scientific Ethical Review Committee Cayetano Heredia Peruvian University, Lima, Peru, 2008-.

14. Jimenez P.: Member Scientific Ethical Review Committee Social Security Mexican Institute, Mexico, 
2006.

15. Lara-Alvarez C.: Member scientific ethical review committee National Institute For Genomic Medicine, Mexico, 2005-2010.

16. Lara-Alvarez C.: Member scientific ethical review committee World Human Genome Organization, 2012-.

17. Maldonado O.: Member Scientific ethical review committee Science Department Javeriana University, 2011-.

18. Melonari P.: Member Scientific ethical review committee Sociedad Argentina Filial Mendoza, Argentina, 2009-.

19. Mercado-Morales E.: Member scientific ethical review committee Hospital Escuela Doctor Roberto Calderon Gutierrez, Nicaragua, 2008-.

20. Mondragon-Barrios L.: Member scientific ethical review committee National Autonomous University of Mexico, 2007; Member Ethics Board National Institute for Genomic Medicine, Mexico, 20072010.

21. Montoya-Montoya G.: Member scientific ethical review committee Medicine Dept. University of Antioquia, Colombia, 2009-.

22. Moreno-Exebio L.: President Scientific Ethical Review Committee National Institute of Health, Lima, Peru, 2005-2010; Member Scientific Ethical Review Committee National Major University San Marcos Faculty of Medicine, Peru, 2007-.

23. Ospina, D.: Member scientific ethics of research committee University of Antioquia, Faculty Nursing, Colombia, 2008-.

24. Rodriguez-Almada H.: Member scientific ethical review committee University Republica, Montevideo, 2010-.

25. Rodriguez-Paz ML.: Member scientific ethical review committee University El Valle, Cali, Colombia, 2010-.

26. Torres MA.: Member Scientific Ethical Review Committee Dentistry Department University of Chile, 2008-.

27. Ulloa-Gonzalez A.: Member Scientific Ethical Review Committee National Autonomous University of Nicaragua, School of Medicine, 2008-.

28. Vidal-Anzardo M.: Member Scientific Ethical Review Committee Cayetano Heredia Peruvian University, Lima, Peru, 2008-.

\section{Participation of trainees in National Bioethics Commissions}

1. Castro JI. Member National Bioethics Commission, Mexico, 2010.

2. Diaz S.: Member National Ethics Commission for Clinical Research, El Salvador, 2007-.

3. Moncayo-Yerovi L.: Member National Bioethics Commission, Ecuador, 2009-.

4. Nieto-Canessa V.: Member National Bioethics Commission, Uruguay, 2010-.

5. Rodriguez-Sanchez K.: Member National Bioethics Committee, Ecuador, 2004-2006.

6. Valencia-Marroquin de Abril H.: Member National ethical review committee, Guatemala, 2009-.

\section{Honors and awards of trainees}

1. Alvarez-Diaz JA.: Manuel Velasco Suarez Bioethics Award, 2007; Member Bioethics National Mexican Academy, 2008.

2. Barrantes-Monge M.: Member Medical Auditing Commission for Clinical Trials, Nicaragua, 2009.

3. Cardozo CA.: Member Editorial Committee Laboratory Animal Latin American Journal, 2009.

4. Gamboa-Bernal G.: Editor Persona y Bioetica, University La Sabana, Colombia, 2006. 
5. Lara-Alvarez C.: President Ethical Commission National Institute for Genomic Medicine, Mexico, 2008-2010.

6. Moreno-Exebio L.: Member National Board for Evaluation of Clinical Essays, Peru, 2006.

7. Vidal-Anzardo M.: First Place Good Governmental Practices for Protection of Human Subjects in Experimental Studies, 2011.

\section{Trainees presentations at Conferences}

1. Alvarez JA. Bioethical debate about the use of human embryos for research. VII National Bioethics Congress, Santiago, Chile, October, 2004).

2. Alvarez JA. Opinions about hypothetical embryo donations of patients participating in assisted reproduction technologies at Latin America. VIII Congress Latin American and Caribbean Federation of Bioethics Institutions, Valparaiso, Chile, June 2011.

3. Ayala CI. Health Justice at Peru. Bioethics congress on ethics in emergency situations, Santiago, Chile, June 2010.

4. Ayala CI. Peruvian vulnerable populations and ethics of research regulations at Peru. Trujillo University, October 2010.

5. Barrantes M. Bioethics and Alzheimer Disease. Alzheimer Foundation of Nicaragua, Managua, June 2006.

6. Barrantes M. Bioethics in Nicaragua. Towards a Subregional Convention on Bioethics and Human Rights, UNESCO FUNGLODE, Santo Domingo, Dominican Republic, February 2007.

7. Barrantes M. Ethical issues in health care for the elderly. Bioethics Simposium, National Autonomous University, Nicaragua, April, 2008.

8. Barrantes M. Ethical issues in subjects with severe Alzheimer. VI Brazilian Bioethics Congress, I MERCOSUR Bioethics Congress and Forum UNESCO Bioethics Net, August-September, 2005.

9. Barrantes M. Ethical Issues on Alzheimer Disease. Inmaculate Conception Laboratory, Managua, Nicaragua, June 2007.

10. Barrantes M. Quality of life and the elderly under an ethical perspective. Health Care Professionals Meeting, University of Chile, Santiago, Chile, October 2005.

11. Borquez B. Adolescence and health care decisions \& Bioethics and security for patients. IV International Congress Army Health and III Nursing, Loja, Ecuador, April, 2007.

12. Borquez B. Bioethics and security of patients. Law contributions: Management of juridical-health risks. VI Brazilian Bioethics Congress, I MERCOSUR Bioethics Congress and Forum UNESCO Bioethics Net, August-September, 2005.

13. Borquez B. Competence versus civil capacity for children in health care decisions. III Conference Bioethics and Law, Pontifitial Catholic University, Santiago, Chile, May 2007.

14. Borquez B. Legal considerationsin health care for the elderly, informed consent. I International Congress Gerontology and Geriatric Nursing Management of Health Care for the Elderly, Valparaiso, Chile, November 2006.

15. Bota A, Estevez A, Fernandez L, Hernandez M, Hevia A, Lara C, Moreno L, Rodriguez K. Scientific Ethical Review Committees. A Pending Challenge in Latin American Countries. VI National Bioethics Congress, Concepcion, Chile, October 2003.

16. Buendia AL. Bioethics as determinant factor in the practice of oral health care professionals (poster and presentation). XVI Oral Health Latin American Seminar - S.O.L.A., II National Students Meeting, Santiago, Chile, August 2004.

17. Buendia AL. Decision-Making in Geriatric and Special Needs Patients. Special Care Denstistry Symposium, Baltimore, USA, April, 2008.

18. Buendia AL. Notions of ethics in geriatric and compromised dental patients. Simposium Geriatric Dentistry, University of Southern California, USA, September, 2008. 
19. Buendia AL. Remedial ethics course - An intervention in Innovations in Medical Education (poster). University of Southern California, U.S.A., March, 2008.

20. Buendia AL. Round Table discussion: Education in Bioethics. I Latin American Meeting on Oral Health Bioethics, University of Chile, November 11-13, 2004.

21. Buendia AL. Tough problems in oral health. University of Southern California Ethics Symposium, University of Southern California, USA, August 2008.

22. Cardozo CA. Divulgation program for the use and care of laboratory animals and ethical review committees for research with animals. Chilean Society of Laboratory Animals Sciences (SOCHI$C A L)$, Santiago, Chile, October 2005.

23. Cardozo CA. Ethics of research with animals. International Meeting Humanism and Bioethics, Fundacion Santa Fe, Bogota, Colombia, October 2010.

24. Cardozo CA. Principles of bioethics in the use of laboratory animals for research and teaching. Chilean Society of Laboratory Animals Sciences (SOCHICAL) and Society of Veterinarians Specialized in Laboratory Animals Sciences ( SOVECAL), Santiago, Chile, October 2005.

25. Cardozo CA. Respect to all forms of life, the animal as subject of rights. VI Brasilean Bioethics Congress, I MERCOSUR Bioethics Congress and Forum UNESCO Bioethics Net, August-September, 2005.

26. Cardozo CA. Towards the conformation of a network for ethical review committees for research with laboratory animals in Latin America. VIII Congress Latin American and Caribbean Federation of Bioethics Institutions, Valparaiso, Chile, June 2011.

27. Cardozo CA. Towards the definition of a national policy for the use and care of laboratory animals. I Meeting National Committees for the use and care of laboratory animals, University Foundation Konrad Lorenz, National University of Colombia, August, 2010.

28. Castro JI. Public Health Insurance as health policy in Mexico to solve the problem of justice in health access for vulnerable populations. Bioethics congress Santiago, Chile Ethics in emergency situations, June 2010.

29. Castro JI. Public Health Insurance in Mexico. II Congress Science, Technology and Culture. Symposium 32 Health Care Service Inequities at Latin America and Caribbean Countries, Santiago, Chile, October 2010.

30. Cosmai NP. Confidentiality and HIV: ethical dilemmas on information disclose. VIII Congress Latin American and Caribbean Federation of Bioethics Institutions, Valparaiso, Chile, June 2011.

31. Diaz E. Ethics of research at Dominican Republic. CONYCIT Dominican Republic, Santo Domingo, April, 2010.

32. Estevez A. Bioethics and Clinical Research. VI National Bioethics Congress, Concepcion, Chile, October 2003.

33. Estevez A. Human rights and ethics of research. Conference Universidad Rio Cuarto, Cordoba, Argentina, June 2007.

34. Estevez A. Responsibility of pharmacologic research in health crisis. Congress Philosophy and Epistemology, National South University, August, 2007.

35. Fernandez L. Informed Consent in Clinical Policies. VI National Bioethics Congress, Concepcion, Chile, October 2003.

36. Gamboa G. Basic bioethics for professionals. Conference Cultural Center Adarve, Bogota, Colombia, July, 2008.

37. Gamboa G. Ethics of scientific publication. Seminar V Anniversary Redalyc, Toluca, Mexico, April, 2008.

38. Gamboa G. Good Clinical Practice and Research. Seminar Hospital Simon Bolivar, Bogota, Colombia, April, 2008.

39. Gamboa G. Quality of life: actual necessity or shadow of consumerism. IV International Family Congress, University La Sabana, Bogota, Colombia, May, 2008.

40. Garbi-Novaes MR, Guilhem D, Lolas F. Ethical actitudes of students and graduates from Medici- 
ne at Brazil with active methodologies and learning based on problem solving. VII Latin American and Caribbean Congress on Bioethics, FELAIBE, Mendoza, June 22, 2009.

41. Garbi-Novaes MR, Guilhem D, Lolas F. Ten years of experience of the scientific ethical review committee of Health Secretary Federal District, Brazil. VII Latin American and Caribbean Congress on Bioethics, FELAIBE, Mendoza, June 22, 2009.

42. Garbi-Novaes MR, Novaes LCG, Guilhem D, Komatsu R, Lolas F. Diagnostic of the humanization, ethics and bioethics insertion into medicine curriculum of Health Sciences School Federal District. Contributions to curriculum management, 46 Brazilian Congress Medical Education, Salvador, Bahia, Brazil, October 2008.

43. Garcia CR. Bioethics in dental practice. Organization III Latin American Simposium on Bioethics in Oral Health, Cayetano Heredia Peruvian University, Lima, Peru, December, 2007.

44. Garcia CR. Bioethics knowledge in relation to oral health practice among dentistry students: preliminary results (poster and presentation). II Venezuelan Congress and II Iberoamerican Congress on Bioethics, Caracas, Venezuela, August 2008.

45. Garcia CR. Knowledge in ethics and bioethics of oral health professionals in teaching (presentation and poster). XXIX International Congress CICAO, Buenos Aires, Argentina, November 2008.

46. Garcia-Perez H. Ethics of research in Mexican-U.S border: current practices and challenges. VIII Congress Latin American and Caribbean Federation of Bioethics Institutions, Valparaiso, Chile, June 2011.

47. Garcia Perez H: The ethics of research on the U.S. -Mexico border: Current practices and challenges. Comparative Border Studies School of Transborder Studies, Arizona State University, March 2012.

48. Jimenez P. Clinical and Research Informed Consent. Catholic University Nordestana (UCNE), San Francisco de Macoris, Dominican Republic, February 2005.

49. Jimenez P. Informed consent in biological sampling. VII National Bioethics Congress, Santiago, Chile, October, 2004.

50. Jimenez P. Introduction to Bioethics and Research Involving Human Beings. INTEC Santo Domingo, Dominican Republic, February 2005.

51. Lara CF. Ethical, legal and social aspects in genomic medicine. National Institute of Genomic Medicine, Mexico, October 2005.

52. Lara CF. Genomic Research and Intellectual Property in the XXI century. International Simposium Humanism and Bioethics. Andes Medical Association, Bogota, Colombia, April 2010.

53. Maldonado O. Bioethics and public health: reflection about child abuse. VIII Congress Latin American and Caribbean Federation of Bioethics Institutions, Valparaiso, Chile, June 2011.

54. Moncayo LP. Ethics of Research in Ecuador. Ministry of Health, Quito, Ecuador, October, 2009.

55. Mondragon L. A genomic medicine for Mexicans: ethical and social implications. Conference Genomic Medicine, National Institute for Genomic Medicine, Mexico, March, 2006.

56. Mondragon L. Bioethics Guide for clinical treatment of patients with suicidal attempt. XXVAnnual Research Meeting National Psychiatric Institute Ramon de la Fuente Muñiz, Mexico, September 2010.

57. Mondragon L. Bioethics, psychology and mental health: care of patients with suicidal attempt. $V I$ Brazilian Bioethics Congress, I MERCOSUR Bioethics Congress and Forum UNESCO Bioethics Net, August-September, 2005.

58. Mondragon L. Conditions of actual ethics for beneficence in physician-patient relationship. XXIII Annual Research Meeting National Psychiatric Institute Ramon de la Fuente Muñiz, Mexico, September 2008.

59. Mondragon L. Ethical aspects of suicide among adolescents. Forum Suicidal Prevention among Adolescents, National Psychiatric Institute Ramon de la Fuente Muñiz, Mexico, Spetember 2010.

60. Mondragon L. Ethical challenges in psychosocial research \& Main ethical dilemmas in research. XXI Annual Research Meeting, National Institute of Psychiatry Ramon de la Fuente, Mexico, October 2006. 
61. Mondragon L. Ethics and human rights. Leadership Training in Mental Health (IMAPACT), Mexico, October 2006.

62. Mondragon L. Ethics and social intervention. Video International Conferences, International Bioethics Net, CAAF and School of Medicine, National Polytechnic Institute, Mexico, April 2006.

63. Mondragon L. Ethics of psychosocial research: Dilemmas and reflections. Fifth World Bioethics Congress, International Society for Bioethics (SIBI), Gijon, Spain, May 2007.

64. Mondragon L. From Episteme to Ethos in Teaching Science. Symposium Science Teaching National Autonomous University, Faculty of Psychology, Mexico, October 2010.

65. Mondragon L. Informed consent: a research task. XXII Annual Research Meeting National Psychiatric Institute Ramon de la Fuente Muñiz, Mexico, September 2007.

66. Mondragon L. Suicide: Right to die? A bioethics approach to the study of suicidal dyadic attempt. II Research Congress Psychology UNAM Psychology Dept., FES-Iztacala y FES Zaragoza, National Autonomous University, Mexico, August, 2008.

67. Mondragon L. The essential place of bioethics in scientific practice. Epistemology, Psychology and Teaching of Science Symposium, National Autonomous University, Mexico, April 2007.

68. Montoya G. Confidentiality and bioethics in the sexual orientation of patients with psychiatric disorders. VII National Bioethics Congress, Santiago, Chile, October, 2004.

69. Moreno L. Development of scientific ethical review committee in Peru - Nacional Bioethis Commissions in Latin America. International Symposium National Health Institute and UNESCO, August, 2010, Lima, Peru.

70. Moreno L. Ethics of Clinical Research. Seminar Workshop at San Marcos National Major University, National Institute of Health, Lima, Peru, October 2005.

71. Moreno L. Ethics of Research. Central Hospital Arm Forces, Lima, Peru, November 2006.

72. Moreno L. Ethics of research. Workshop to National Public Health Laboratories Net at National Institute of Health, Lima, Peru, November 2005.

73. Moreno L. Health research priorities at Peru. III Videoconference Health Priority \& Intercultural Health, CIEB, Santiago, Chile, July 2007.

74. Nieto V. Bioethics training course for Public Health Institute health management staff. VIII Congress Latin American and Caribbean Federation of Bioethics Institutions, Valparaiso, Chile, June 2011.

75. Nieto V. Ethical challenges in the process of decision making about fetus with pathology. Perspectives of health care team. VIII Congress Latin American and Caribbean Federation of Bioethics Institutions, Valparaiso, Chile, June 2011.

76. Nieto V. Health Reform at Uruguay Republic and social inequities. II Congress Science, Technology and Culture. Simposium 32 Health Care Service Inequities at Latin America and Caribbean Countries, Santiago, Chile, October 2010.

77. Nieto V. Health Reform at Uruguay Republic. Bioethics congress Ethics in emergency situations, Santiago, Chile, June 2010.

78. Ormaeche M. ¿̇s inequity damaging the health of indigenous populations? VI Brazilian Bioethics Congress, I MERCOSUR Bioethics Congress and Forum UNESCO Bioethics Net, August-September, 2005.

79. Pavon S. Health justice at Argentina. Bioethics congress ethics in emergency situations, Santiago, Chile, June 2010.

80. Pavon S. Coordination Therapeutics for Sleep Medicine. III Chilean Congress on Sleep Medicine, Chilean Society for Sleep Medicine (SOCHIMES), Santiago, Chile, July 2010.

81. Pavon S. Health care inequities at Argentina. II Congress Science, Technology and Culture. Simposium 32 Health Care Service Inequities at Latin America and Caribbean Countries, October 2010, Santiago, Chile.

82. Rendon AE. Innovation in biotechnology in developing countries. Agrobiotechnology Congress, Corrientes, Argentina, October 2006. 
83. Rendon AE. Intelectual property and biotechnology at Latin America. Situation, challenges and possibilities. Bicentennial Program in Science and Technology Ethics and Innovation Technology. Towards a Social Dialogue in the Hidden Dimension of Knowledge Transfer, organized by CIEB University of Chile, with the collaboration of Pharmaceutic Industry Chamber and CONICYT, November, 2006.

84. Rendon AE. Risks and opportunities in intelectual property of genetic material at Latin America. II National Congress on Genomic Medicine INMEGEN, October, 2006.

85. Rodriguez K. Bioethical principles in Medical Auditing. ANACSE, Guayaquil, Ecuador, July 2005.

86. Rodriguez K. CIOMS Ethical Guidelines. Tungurahua Medical Conference, Tungurahua, Ecuador, March, 2005.

87. Rodriguez K. Confidentiality in Medical practice. Metromedical Seminar, Quito, Ecuador, October 2005.

88. Rodriguez K. Coordinator Bioethics IV Congress Latin American Society for Internal Medicine, SOLAMI, Quito, Ecuador, May 2005.

89. Rodriguez K. Ethics in the Clinical Laboratory. Informed consent: resource for medical protection or tool for safeguarding the subject?, Ecuadorian Medical Association, Quito, Ecuador, September 2005.

90. Rodriguez K. Ethics of Placebo use. ASOPROFAR, Quito, Ecuador, February 2005.

91. Rodriguez K. II International Congress of Laboratory Medicine and V Ecuadorian Congress of Clinical Pathology and Laboratory Medicine, Quito, Ecuador, July 2005.

92. Rodriguez K. Models for physician-patient relationship under a bioethical perspective. IV Latin American Congress of Internal Medicine, Latin American Society of Internal Medicine, Quito, Ecuador, May 2005.

93. Rodriguez K. Perception about bioethical issues in health care by users of Health Services at QuitoEcuador. Physician post degree USFQ 2009-2010. VIII Congress Latin American and Caribbean Federation of Bioethics Institutions, Valparaiso, Chile, June 2011.

94. Rodriguez K. Scientific ethical review committees. International Seminar for the Ethics of Research, Pontifical Catholic University, Ecuador, May 2005.

95. Rodriguez ML. Health Justice at Colombia. Bioethics congress Santiago, Chile on ethics in emergency situations, June 2010.

96. Sanders-Bruletti MM. Reflections about teaching bioethics. VIII Congress Latin American and Caribbean Federation of Bioethics Institutions, Valparaiso, Chile, June 2011.

97. Torres MA. Oral Rehabilitation Seminar: Bioethical Dilemmas in Ethical Rehabilitation. Prothesis and Oral Rehabilitation Society, Santiago, Chile, June 2007.

98. Torres MA. Round Table discussion: Ethics in professional practice. I Latin American Meeting on Oral Health Bioethics, University of Chile, November 11-13, 2004.

99. Torres-Quintana MA, Cortes AJ, Miranda VM, Urzua OB, Fajreldin V. Scientific ethical review committee of Dentistry Department University of Chile. Critical review. VIII Congress Latin American and Caribbean Federation of Bioethics Institutions, Valparaiso, Chile, June 2011.

100. Ulloa A. Bioehics philosophical basis and basic issues. Forum Medical Ethics, National Autonomous University, December 2008.

101. Ulloa A. Bioethics training and organization of scientific ethical review committees. III Simposium Medical Ethics, National Autonomous University, Nicaragua, September, 2008.

102. Ulloa A. Informed consent in surgery interventions. Hospital Antonio Lenin Fonseca, Nicaragua, April, 2008.

103. Valencia H. Ethics of research at Guatemala. National University San Carlos, Guatemala, April, 2010.

104. Vidal M. Family discrimination of patients with HIV-AIDS as violating non maleficence. VI Brazilian Bioethics Congress, I MERCOSUR Bioethics Congress and Forum UNESCO Bioethics Net, AugustSeptember 2005. 


\section{APPENDIX II}

\section{Faculty publications related to the program (selected)}

\section{Books}

1. Cardozo C, Lolas F, Quezada A, Rodriguez E. (editores) Etica y Odontologia. Una Introduccion. Santiago de Chile: Programa de Bioetica OPS/OMS, CIEB Universidad de Chile; 2007.

2. Cardozo CA, Mrad de Osorio A, Martinez C, Rodriguez E, Lolas F. El animal como sujeto experimental. Aspectos tecnicos y eticos. Santiago de Chile: Programa de Bioetica OPS/OMS, CIEB, Universidad de Chile, 2007.

3. CIOMS. Pautas eticas internacionales para la investigacion biomedica en seres humanos CIOMS 2002. Traduccion del PRB /OPS/OMS. Santiago de Chile: Programa Regional de Bioetica OPS/ OMS; 2003.

4. Escribar A, Perez M, Villarroel R. (editores) Bioetica: fundamentos y dimension practica. Santiago de Chile: Mediterraneo; 2004.

5. Leon FJ, Arratia A, Casas ML, Rodriguez E. Bioetica general y clinica. Santiago de Chile: Fundacion Interamericana Ciencia y Vida; 2010.

6. Lolas F, Drumond JG de Freitas. Fundamentos de uma antropologia bioetica. O apropiado, o bom e o justo. Sao Paulo, Brasil: Edicoes Loyola, Centro Universitario Sao Camilo; 2007.

7. Lolas F, Drumond, JG de Freitas. Etica em Engenharia e Tecnologia. Brasilia, Brasil: Conselho Federal de Engenharia, Arquitetura e Agrononia (Confea); 2011.

8. Lolas F, Martin D, Quezada A. (editores) Prioridades en salud y salud intercultural. Estudios de bioetica social No 1. Santiago de Chile: Programa de Bioetica OPS/OMS, CIEB Universidad de Chile; 2007.

9. Lolas F, Quezada A, Rodriguez E. (editores) Investigacion en Salud. Dimension Etica. Santiago de Chile: Programa de Bioetica OPS/OMS, CIEB University of Chile; 2006.

10. Lolas F, Quezada A. (editores) Pautas eticas de investigacion en sujetos humanos: nuevas perspectivas. Santiago de Chile: Programa Regional de Bioetica OPS/OMS; 2003.

11. Lolas F. (editor) Etica e Innovacion Tecnologica. Santiago de Chile: Programa de Bioetica OPS/ OMS, CIEB University of Chile; 2007.

12. Lolas F. (editor) Dialogo y cooperacion en salud. Diez años de bioetica en la OPS. Santiago de Chile: Unidad de Bioetica OPS/OMS; 2004.

13. Lolas F. Bioetica en America Latina. Una decada de evolucion. Monografia de Acta Bioethica № 4. Santiago de Chile: CIEB Universidad de Chile; Programa de Bioetica OPS/OMS, 2010.

14. Pessini L, De Barchifontaine C, Lolas F. (coordinadores) Perspectivas de la bioetica en Iberoamerica. Santiago de Chile: Programa de Bioetica OPS/OMS; 2007.

15. Pessini L, De Barchifontaine C, Lolas F. Iberoamerican Bioethics. History and Perspectives. Heidelberg: Springer Dordrecht; 2010.

In preparation: Etica de la Investigacion Biomedica y Psicosocial, Lolas F. Rodriguez E., Quezada A. (editors). Centro Interdisicplinario de Estudios en Bioetica, Universidad de Chile, 2011.

\section{Chapters of books}

1. Aguila A, Lagos K. Actualizacion de registros de comites de etica y regulaciones en paises de Latinoamerica y el Caribe. En: Lolas F. (editor) Dimensiones eticas de las regulaciones en salud. Monografia de Acta Bioethica No 3. Santiago de Chile: CIEB Universidad de Chile, Programa de 
Bioetica OPS/OMS; 2009: 403-411.

2. Aguila A. Recursos de informacion para la investigacion. En: Lolas F, Quezada A, Rodriguez E. (editores) Investigacion en Salud. Dimension Etica. Santiago de Chile: Programa de Bioetica OPS/ OMS, CIEB University of Chile; 2006: 293-303.

3. Goodman KW. Health priorities, conflict of interest and vulnerable populations: how ethics precedes law in the protection of human subjects. En: Lolas F. (editor) Dimensiones eticas de las regulaciones en salud. Monografia de Acta Bioethica No 3. Santiago de Chile: CIEB Universidad de Chile, Programa de Bioetica OPS/OMS; 2009: 221-232.

4. Hickling FW, Lolas F, Sorel E, Hutchinson G. Ethical issues in psychiatry: A Caribbean perspective. In: Hickling FW, Sorel E. (editors) Images of Psychiatry: The Caribbean. Kingston: University of the West Indies; 2005: 361-379.

5. Iribarra R, Rodriguez E, Torres MA. Bioetica, bioseguridad y responsabilidad social: nuevo campo de reflexion para el odontologo. En: Cardozo C, Lolas F, Quezada A, Rodriguez E. (editores) Etica y Odontologia. Una Introduccion. Santiago de Chile: Programa de Bioetica OPS/OMS, CIEB Universidad de Chile; 2007: 129-138.

6. Lagos K, Rodriguez E. Ciencia e Investigacion. En: Lolas F, Quezada A, Rodriguez E. (editores) Investigacion en Salud. Dimension Etica. Santiago de Chile: Programa de Bioetica OPS/OMS, CIEB University of Chile; 2006: 25-38.

7. Litewka S. America Latina: multiculturalidad y regulaciones en etica de la investigacion. En: Lolas F. (editor) Dimensiones eticas de las regulaciones en salud. Monografia de Acta Bioethica No 3. Santiago de Chile: CIEB Universidad de Chile, Programa de Bioetica OPS/OMS; 2009: 243-260.

8. Lolas F, Outomuro D. Bioetica y salud publica. En: Lolas F, Quezada A, Rodriguez E. (editores) Investigacion en Salud. Dimension Etica. Santiago de Chile: Programa de Bioetica OPS/OMS, CIEB University of Chile; 2006: 103-115.

9. Lolas F, Outomuro D. Etica en la publicacion de los resultados de la investigacion. En: Lolas F, Quezada A, Rodriguez E. (editores) Investigacion en Salud. Dimension Etica. Santiago de Chile: Programa de Bioetica OPS/OMS, CIEB University of Chile; 2006: 259-272.

10. Lolas F, Rodriguez E. Conflictos de interes en la investigacion biomedica y psicosocial. En: Lolas F, Quezada A, Rodriguez E. (editores) Investigacion en Salud. Dimension Etica. Santiago de Chile: Programa de Bioetica OPS/OMS, CIEB University of Chile; 2006: 231-238).

11. Lolas F. A Organizacao Pan-Americana da Saude (PAHO) e a bioetica na America Latina. En: Pessini L, De Barchifontaine P. Bioetica na Ibero-America: Historia e Perspectivas. Sao Paulo: Sao Camilo y Edicoes Loyola; 2007: 55-62.

12. Lolas F. Apuntes sobre bioetica en America Latina. En: Organizacion Panamericana de la Salud (OPS/OMS). La equidad en la mira: la salud publica en Ecuador durante las ultimas decadas. Quito: OPS/MSP/CONASA; 2007: 407-413.

13. Lolas F. Bioethical sustainability. Towards a value-based epistemic community in the life sciences and healthcare. In: UNESCO. National Bioethics Committees in action. Paris: UNESCO; 2010: 113-115.

14. Lolas F. Bioetica y salud en las Americas y el Caribe: cooperacion y dialogo en el establecimiento de prioridades. En: Lolas F, Martin D, Quezada A. (editores) Prioridades en salud y salud intercultural. Santiago de Chile: Programa de Bioetica OPS/OMS, CIEB Universidad de Chile; 2007: 71-81.

15. Lolas F. Consideraciones sobre la bioetica en Latinoamerica. En: Campbell U, Alvarez Diaz JA. (coordinadores) Bioetica en perspectiva. Mexico: Universidad Autonoma de Ciudad Juarez; 2008: 157-173.

16. Lolas F. De fisiologia, psiquiatria y bioetica. Algunas precisiones personales e incompletas sobre mis trabajos. En: Fuller A. (editora) Huella y Presencia. Tomo VII. Santiago de Chile: Universidad de Chile, Facultad de Medicina; 2005: 211-221. 
17. Lolas F. De genomica e genetica: Bionomica e Bioetica. En: Romeo-Casabona C, Freire de Sa M. (coordinadores) Desafios Juridicos da Biotecnologia. Belo Horizonte, Brasil: Mandamentos Editora; 2007: 65-76.

18. Lolas F. De la praxis al texto, del texto a la praxis. La bioetica como hermeneutica. En: Lolas F. (editor) Dimensiones eticas de las regulaciones en salud. Monografia de Acta Bioethica No 3. Santiago de Chile: CIEB Universidad de Chile, Programa de Bioetica OPS/OMS; 2009: 29-42.

19. Lolas F. Dimension bioetica de las profesiones. En: Lolas F. Bioetica en America Latina. Una decada de evolucion. Monografia de Acta Bioethica No 4. Santiago de Chile: CIEB Universidad de Chile, Programa de Bioetica OPS/OMS; 2010: 139-158.

20. Lolas F. El caso Balmis y la etica de la vacunacion. Una nota historica. En: Lolas F. Bioetica en America Latina. Una decada de evolucion. Monografia de Acta Bioethica No 4. Santiago de Chile: CIEB Universidad de Chile, Programa de Bioetica OPS/OMS; 2010: 159-164.

21. Lolas F. El caso Cajal y el español como lengua cientifica. En: Lolas F. Bioetica en America Latina. Una decada de evolucion. Monografia de Acta Bioethica No 4. Santiago de Chile: CIEB Universidad de Chile, Programa de Bioetica OPS/OMS; 2010: 165-170.

22. Lolas F. El desafio bioetico de la equidad: su relevancia en salud publica. En: Malaga H. Salud Publica. Enfoque bioetico. Caracas: Disinlimed; 2005: 35-40.

23. Lolas F. El discurso disciplinario: una nota sobre sus atributos. En: Harvey A. (compiladora) En torno al discurso. Contribuciones de America Latina. Santiago de Chile: Ediciones Universidad Catolica de Chile; 2005: 329-338.

24. Lolas F. El imperativo bioetico de Fritz Jahr y la neobioetica estadounidense. En: Lolas F. Bioetica en America Latina. Una decada de evolucion. Monografia de Acta Bioethica No 4. Santiago de Chile: CIEB Universidad de Chile, Programa de Bioetica OPS/OMS; 2010: 171-176.

25. Lolas F. El lugar de la verdad en un mundo de evidencias: la retorica de las ciencias biomedicas. En: Grammatico G, Arbea A. (editores) Quid est Veritas? Ensayos. Coleccion ITER, vol. XIII; 2005: 175-181.

26. Lolas F. Estudios de bioetica social. Prioridades en salud y salud intercultural. En: Lolas F, Martin D, Quezada A. (editores) Prioridades en salud y salud intercultural. Santiago de Chile: Programa de Bioetica OPS/OMS, CIEB Universidad de Chile; 2007: 15-27.

27. Lolas F. Ethics of preparedness in Latin America. Considerations on global epidemic risks. En: Lolas F, Martin D, Quezada A. (editores) Prioridades en salud y salud intercultural. Santiago de Chile: Programa de Bioetica OPS/OMS, CIEB Universidad de Chile; 2007: 105-112.

28. Lolas F. Etica de la innovacion tecnologica en medicina. En: Lolas F. (editor) Etica e innovacion tecnologica. Santiago de Chile: Programa de Bioetica OPS/OMS, CIEB Universidad de Chile; 2006: 165-178).

29. Lolas F. Etica y ensayos clinicos: preguntas y dilemas. En: Garbi Novaes MR, Lolas F, Quezada A. Etica y Farmacia. Una perspectiva latinoamericana. Monografia de Acta Bioethica No 2. Santiago de Chile: CIEB Universidad de Chile, Programa de Bioetica OPS/OMS; 2009: 199-210.

30. Lolas F. Evolucion y transformaciones en el ejercicio de la profesion medica. En: Profesionalismo medico en el actual escenario de la salud. Santiago de Chile: Asociacion de Medicos de la Clinica Alemana AMCA; 2008: 29-37.

31. Lolas F. Funcion de los comites de etica de la investigacion. Una respuesta en busca de preguntas. En: Lolas F. (editor) Etica e innovacion tecnologica. Santiago de Chile: Programa de Bioetica OPS/ OMS, CIEB Universidad de Chile; 2006: 217-221.

32. Lolas F. Genomica e bioetica: o valor da ciencia na sociedade. En: Garrafa V, Pessini L. (organizadores) Bioetica: Poder e Injustica. Sao Paulo, Brasil: Edicoes Loyola \& Centro Universitario Sao Camilo; 2003: 129-136.

33. Lolas F. Genomica y bioetica: culturas epistemicos y construccion social. En: Brena I, Diaz Muller LT. (coordinadores) Segundas jornadas sobre globalizacion y derechos humanos: bioetica y biotecnolo- 
gia. Mexico: Instituto de Investigaciones Juridicas, Universidad Nacional Autonoma de Mexico; 2004: 71-90.

34. Lolas F. Genomica y bioetica. Culturas epistemicas y construccion social. En: Lolas F. (editor) Etica e innovacion tecnologica. Santiago de Chile: Programa de Bioetica OPS/OMS, CIEB Universidad de Chile; 2006: 101-114.

35. Lolas F. Investigacion versus intervencion en salud publica: ¿Una distincion etica? En: Lolas F, Martin D, Quezada A. (editores) Prioridades en salud y salud intercultural. Santiago de Chile: Programa de Bioetica OPS/OMS, CIEB Universidad de Chile; 2007: 39-47).

36. Lolas F. La bioetica como filosofia social en la formulacion de politicas publicas. Consideraciones sobre epistemologia, sustentabilidad y sostenibilidad. En: Fundacion Fernando Fueyo Laneri (editores). Estudios de Derecho Privado. Santiago de Chile: Editorial Juridica de Chile; 2008: 607-615.

37. Lolas F. La clonacion de humanos: aproximacion etica. En: Fundacion Fernando Fueyo Laneri. Derecho, Bioetica y Genoma Humano. Santiago de Chile: Editorial Juridica de Chile; 2003: 145156.

38. Lolas F. La dimension bioetica de la psiquiatria. En: Marchant N, Monchablon A. (editores) Tratado de Psiquiatria. Buenos Aires, Argentina: Grupo Guia S.A.; 2005: 1905-1912.

39. Lolas F. La dimension etica de la farmacologia y la farmacia. En: Garbi Novaes MR, Lolas F, Quezada A. Etica y Farmacia. Una perspectiva latinoamericana. Monografia de Acta Bioethica No 2. Santiago de Chile: CIEB Universidad de Chile, Programa de Bioetica OPS/OMS; 2009: 19-22.

40. Lolas F. La innovacion como tarea social. El ejemplo de las tecnologias de la representacionrepresentatividad. En: Lolas F. (editor) Etica e innovacion tecnologica. Santiago de Chile: Programa de Bioetica OPS/OMS, CIEB Universidad de Chile; 2006: 191-208.

41. Lolas F. La investigacion con animales. Una bioetica para la ciencia de fronteras. En: Cardozo C, Mrad de Osorio A, Martinez C, Rodriguez E, Lolas F. (editores) El animal como sujeto experimental. Aspectos tecnicos y eticos. Santiago de Chile: Programa de Bioetica OPS/OMS, CIEB Universidad de Chile; 2007: 13-19.

42. Lolas F. La Organizacion Panamericana de la Salud y la bioetica. En: Pessini L, De Barchifontaine C, Lolas F. Perspectivas de la bioetica en Iberoamerica. Santiago de Chile: Programa de Bioetica OPS/OMS, CIEB Universidad de Chile, Sao Camilo; 2007: 45-50.

43. Lolas F. La reforma del sector salud y los fines de la medicina. En: Malaga H. Salud Publica. Enfoque bioetico. Caracas: Disinlimed; 2005: 81-86.

44. Lolas F. Macrobioetica y el proceso de construccion de la salud. En: Malaga H. Salud Publica. Enfoque bioetico. Caracas: Disinlimed; 2005: 137-144.

45. Lolas F. Medicina Antropologica y Bioetica. Viktor Von Weizsacker sobre el Juicio de Nurenberg. En: Lolas F. La Medicina Antropologica y el Juicio de Nurenberg. El aporte de Viktor Von Weizsacker. Monografia de Acta Bioethica No 5. Santiago de Chile: CIEB Universidad de Chile; 2010: 39-62.

46. Lolas F. Modelos de calidad de vida. En: Agenda Puertorriqueña para la Calidad de Vida. San Juan, Puerto Rico: Fundacion Operacion Solidaridad; 2006: 29-37.

47. Lolas F. Principios bioeticos y calidad de la atencion medica. En: Lolas F. (editor) Etica e innovacion tecnologica. Santiago de Chile: Programa de Bioetica OPS/OMS, CIEB Universidad de Chile; 2006: 209-216.

48. Lolas F. Psiquiatria y etica. En: Alarcon R, Mazzotti G, Nicolini H. (editores) Psiquiatria. Mexico: El Manual Moderno y Organizacion Panamericana de la Salud; 2004: 945-951.

49. Lolas F. Sobre o ethos da medicina. Uma desposta a Jose Geraldo de Freitas Drumond. En: Drumond G. de Freitas. O Ethos Medico. A velha e a nova moral medica. Montes Claros, MG: Editora Unimontes; 2005: XXXVII-XLVI.

50. Lolas F. Tecnologias sanitarias en el contexto social: una reflexion bioetica. En: Lolas F. (editor) Etica e innovacion tecnologica. Santiago de Chile: Programa de Bioetica OPS/OMS, CIEB Universidad de Chile; 2006: 179-190. 
51. Lolas F. The Pan American Health Organization and Latin American Bioethics. In: Pessini L, De Barchifontaine C, Lolas F. (editors) Iberoamerican Bioethic. History and Perspectives. Heidelberg: Springer Dordrecht; 2010: 55-62.

52. Lolas F. Towards a value-based public health in Latin America and the Caribbean. The work of PAHO bioethics program. En: Lolas F. (editor) Dimensiones eticas de las regulaciones en salud. Monografia de Acta Bioethica No 3. Santiago de Chile: CIEB Universidad de Chile, Programa de Bioetica OPS/OMS; 2009: 389-402.

53. Lolas F. Uno de los juicios en Nurenberg. Los medicos y la ciencia medica”. En: Lolas F. La Medicina Antropologica y el Juicio de Nurenberg. El aporte de Viktor Von Weizsacker. Monografia de Acta Bioethica No 5. Santiago de Chile: CIEB Universidad de Chile; 2010: 29-38.

54. Lolas F. Viktor Von Weizsacker: Vida y obra. En: Lolas F. La Medicina Antropologica y el Juicio de Nurenberg. El aporte de Viktor Von Weizsacker. Monografia de Acta Bioethica No 5. Santiago de Chile: CIEB Universidad de Chile; 2010: 13-28.

55. Lolas, F. Bipersonalidad, un constructo de la Escuela de Heidelberg. En: Lolas F. Bioetica en America Latina. Una decada de evolucion. Monografia de Acta Bioethica No 4. Santiago de Chile: CIEB Universidad de Chile, Programa de Bioetica OPS/OMS; 2010: 177-184.

56. Mancini R. Formacion en Bioetica en America Latina y el Caribe. En: Lolas F. (editor) Dialogo y Cooperacion en Salud. Diez Años de Bioetica en la OPS. Santiago de Chile: Unidad de Bioetica OPS/OMS; 2004: 161-172.

57. Pessini L, Saad-Hossne W. Ethics of research with humans in Brazil. En: Lolas F. (editor) Dimensiones eticas de las regulaciones en salud. Monografia de Acta Bioethica No 3. Santiago de Chile: CIEB Universidad de Chile, Programa de Bioetica OPS/OMS; 2009: 173-178.

58. Quezada A. Perspectivas eticas de la publicacion biomedica. En: Garbi Novaes MR, Lolas F, Quezada A. Etica y Farmacia. Una perspectiva latinoamericana. Monografia de Acta Bioethica No 2. Santiago de Chile: CIEB Universidad de Chile, Programa de Bioetica OPS/OMS; 2009: 301-312.

59. Quezada A. Viktor Von Weizsacker y otro espiritu para la Medicina. En: Lolas F. La Medicina Antropologica y el Juicio de Nurenberg. El aporte de Viktor Von Weizsacker. Monografia de Acta Bioethica No 5. Santiago de Chile: CIEB Universidad de Chile; 2010: 197-206.

60. Rodriguez E, Lolas F. Social Issues Related to Gene Patenting in Latin America: A Bioethical Reflection. In: Hongladarom S. (editor) Genomics and Bioethics: Interdisciplinary Perspectives, Technologies and Advancements. New York: Medical Information Science Reference; November 2010: 151-170.

61. Rodriguez E, Outomuro D. Etica de los ensayos clinicos. En: Lolas F, Quezada A, Rodriguez E. (editores) Investigacion en Salud. Dimension Etica. Santiago de Chile: Programa de Bioetica OPS/ OMS, CIEB University of Chile; 2006: 151-176.

62. Rodriguez E. Bases bioeticas de las regulaciones sanitarias. En: Lolas F. (editor) Dimensiones eticas de las regulaciones en salud. Monografia de Acta Bioethica No 3. Santiago de Chile: CIEB Universidad de Chile, Programa de Bioetica OPS/OMS; 2009: 85-101.

63. Rodriguez E. Bioetica y Cristianismo En: Campbell Manjarrez U. (editor) Bioetica en la frontera. Ciudad Juarez: UACJ; 2006.

64. Rodriguez E. Conflictos de interes en investigacion y comites de etica. En: Leon FJ. (coord.) Etica Clinica y Comites de Etica en Latinoamerica. Santiago de Chile: Felaibe y Fundacion Ciencia y Vida; 2011: 33-38.

65. Rodriguez E. Critica de Viktor Von Weizsacker a la eutanasia y a la idea de sujeto como objeto de investigacion. En: Lolas F. La Medicina Antropologica y el Juicio de Nurenberg. El aporte de Viktor Von Weizsacker. Monografia de Acta Bioethica No 5. Santiago de Chile: CIEB Universidad de Chile; 2010: 189-196.

66. Rodriguez E. Desarrollo e innovacion tecnologica. Etica de los fines y medios. En: Lolas F. (editor) Etica e innovacion tecnologica. Santiago de Chile: Programa de Bioetica OPS/OMS, CIEB Universidad de Chile; 2006: 35-48. 
67. Rodriguez E. El consentimiento informado en el uso de muestras biologicas humanas y de registros medicos. Comentario a las Pautas Eticas Internacionales para la Investigacion Biomedica en Seres Humanos CIOMS 2002. En: Lolas F, Quezada A. (editores) Pautas eticas de investigacion en sujetos humanos: nuevas perspectivas. Santiago de Chile: Programa Regional de Bioetica OPS/ OMS; 2003: 45-54.

68. Rodriguez E. Eleccion de control en ensayos clinicos. El problema etico del uso de placebo. Comentario a las Pautas Eticas Internacionales para la Investigacion Biomedica en Seres Humanos CIOMS 2002. En: Lolas F, Quezada A. (editores) Pautas eticas de investigacion en sujetos humanos: nuevas perspectivas. Santiago de Chile: Programa Regional de Bioetica OPS/OMS; 2003: 55-64.

69. Rodriguez E. Etica de la investigacion con embriones humanos. En: Lolas F, Quezada A, Rodriguez E. (editores) Investigacion en Salud. Dimension Etica. Santiago de Chile: Programa de Bioetica OPS/OMS, CIEB University of Chile; 2006: 177-189.

70. Rodriguez E. Etica de la investigacion en genetica humana. En: Lolas F, Quezada A, Rodriguez E. (editores) Investigacion en Salud. Dimension Etica. Santiago de Chile: Programa de Bioetica OPS/ OMS, CIEB University of Chile; 2006: 117-150.

71. Rodriguez E. Etica en innovacion tecnologica y farmacogenomica. En: Garbi Novaes MR, Lolas F, Quezada A. Etica y Farmacia. Una perspectiva latinoamericana. Monografia de Acta Bioethica No 2. Santiago de Chile: CIEB Universidad de Chile, Programa de Bioetica OPS/OMS; 2009: 265-282.

72. Rodriguez E. Innovacion tecnologica y genoetica. En: Lolas F. (editor) Etica e innovacion tecnologica. Santiago de Chile: Programa de Bioetica OPS/OMS, CIEB Universidad de Chile; 2006: 85-99.

73. Rodriguez E. Principios Bioeticos en Salud Publica. En: Leon FJ. (coordinador) Etica y Justicia Sanitaria en Situaciones de Catastrofe. Santiago de Chile: Sociedad Chilena de Bioetica; 2010: 33-38.

74. Rodriguez E. Relacion medico-paciente y la practica medica. En: Lolas F. (editor) Dialogo y Cooperacion en Salud. Diez Años de Bioetica en la OPS. Santiago de Chile: Unidad de Bioetica OPS/ OMS; 2004: 61-729.

75. Rodriguez E., Moreno L. Los principios eticos y la conduccion responsable de la investigacion. En: Lolas F, Quezada A, Rodriguez E. (editores) Investigacion en Salud. Dimension Etica. Santiago de Chile: Programa de Bioetica OPS/OMS, CIEB University of Chile; 2006: 279-292.

76. Rueda L. Etica e investigacion en seres humanos. En: Escribar A, Perez M, Villarroel R. (editores) Bioetica: fundamentos y dimension practica. Santiago de Chile: Mediterraneo; 2004.

77. Sorel E, Lolas F. PAHO bioethics, public health and health policy forum. En: Lolas F, Martin D, Quezada A. (editores) Prioridades en salud y salud intercultural. Santiago de Chile: Programa de Bioetica OPS/OMS, CIEB Universidad de Chile; 2007: 187-189.

78. Valdebenito C. Antropologia medica: aproximacion a la salud oral. En Cardozo C, Lolas F, Quezada A, Rodriguez E. (editores) Etica y Odontologia. Una Introduccion. Santiago de Chile: Programa de Bioetica OPS/OMS, CIEB Universidad de Chile; 2007: 53-57.

\section{Journal articles}

1. Ferrer JJ. La bioetica como quehacer filosofico. Acta Bioethica 2009; 15(1): 35-41. NIHMS\# 132502.

2. Herrera S, Rodriguez E. Etnoconocimiento en Latinoamerica. Apropiacion de recursos geneticos y bioetica. Acta Bioethica 2004; 10(2): 181-190.

3. Lolas F, Rodriguez E, Valdebenito C. El proyecto del genoma en la literatura biomedica latinoamericana de cuatro paises. Acta Bioethica 2004; 10(2): 167-180.

4. Lolas F. Bioethics and animal research. A personal perspective and a note on the contribution of 
Fritz Jahr. Biological Research (Santiago) 2008; 41: 119-123. NIHMS\# 247396.

5. Lolas F. Bioethics at the Pan American Health Organization: Origins, developments, and challenges. Acta Bioethica 2006; 12(1): 113-119.

6. Lolas F. Comites de etica de la investigacion: una solucion en busca de problemas. Revista Chilena de Reumatologia 2007; 23(1): 2-4.

7. Lolas F. Conflictos de interes en la investigacion clinica y el trabajo asistencial. Revista de Psiquiatria Clinica (Santiago de Chile) 2005; 42(2): 6-9.

8. Lolas F. Consideraciones sobre vulnerabilidad social. Quiron (La Plata) 2004; 35: 45-51.

9. Lolas F. Defensa de la biodiversidad. Societas (Academia Chilena de Ciencias Sociales, Politicas y Morales) 2011; 13: 73-75.

10. Lolas F. El sujeto de investigacion: Aproximaciones a su estudio. Persona (Lima) 2005; 8: 59-67.

11. Lolas F. Investigacion que involucra sujetos humanos: dimensiones tecnicas y eticas. Acta Bioethica 2004; 10(1): 11-16.

12. Lolas F. Medicina antropologica y bioetica. Viktor von Weizsäcker sobre el juicio de Nürenberg. Archivos de Psiquiatria (Madrid) 2008; 70(4): 267-282.

13. Lolas F. Outomuro D. Bioetica y salud publica. Bioetica Selecciones, CENALBE, abril 2007; 11: 107-120.

14. Lolas F. Psiquiatria y bioetica. Monografias de Psiquiatria, Aula Medica, Madrid 2003; 15(3): 1-26.

15. Lolas F. Public health and social justice. Toward ethical sustainability in healthcare and research. Acta Bioethica 2003; 9(2): 189-194.

16. Lolas F. Rehistoriar la bioetica en Latinoamerica. La contribucion de James Drane. Acta Bioethica 2005; 11(2): 161-167.

17. Lolas F. Salud mental y psiquiatria: pluralidad y hetrogeneidad. Revista Chilena de Neuro-Psiquiatria (Santiago) 2008; 46(2): 97-98.

18. Lolas F. Salud publica. ¿Practica versus investigacion? Investigacion en Salud (Guadalajara) 2005; 7: $4-5$.

19. Rodriguez E, Lolas F. The topic of research integrity in Latin America. Bioethikos 2011; 5(4): 362368. PMCID\# 360678.

20. Rodriguez E, Valdebenito C, Kanner E, Lolas F. El proyecto del genoma humano y las regulaciones juridicas en cuatro paises latinoamericanos. Jurisprudencia Argentina 2004; 4, Numero Especial Bioetica, fasciculo No 5: 42-50.

21. Rodriguez E, Valdebenito C, Lolas F. Depresion y estigma social. Revista de Psiquiatria Clinica (Santiago) 2004; 41(2): 43-48.

22. Rodriguez E, Valdebenito C, Misseroni A, Fernandez L, Outomuro D, Schiattino I, Lolas F. Percepciones Sociales sobre Genomica en Cuatro Paises Latinoamericanos. Implicaciones Etico Legales. Derecho y Genoma 2004; 21: 141-164.

23. Rodriguez E, Valdebenito C, Misseroni A, Fernandez L, Outomuro D, Schiattino I, Ferrer M, Lolas F. Social, ethical and legal attitudes towards genomic research in four Latin American countries. Electronic Journal of Biotechnology December 2005; 8(3). Available from: http://www.ejbiotechnology.info/content/vol8/issue3/full/9/index.html. ISSN 0717-3458.

24. Rodriguez E. Comites de Evaluacion Etica y Cientifica para la Investigacion en Seres Humanos y las Pautas CIOMS 2002. Acta Bioethica 2004; 10(1): 37-48.

25. Rodriguez E. Cultura etica e investigacion en salud. Acta Bioethica 2005; 11(1): 11-22.

26. Rodriguez E. Etica de la investigacion en modelos animales de enfermedades humanas. Acta Bioethica 2007; 13(1): 25-40.

27. Rodriguez E. Temas para una bioetica latinoamericana. Acta Bioethica 2009; 15(1): 87-93. NIHMS\# 132494.

28. Rodriguez E. Terapia genica y principios eticos. Acta Bioethica 2003; 9(1): 69-80.

29. Schiattino I, Silva C, Lolas F, Valdebenito C, Rodriguez E. Descripcion de las percepciones sobre 
el proyecto genoma humana en Chile, Peru, Argentina y Mexico. Quiron 2005; 36(1/3).

30. Schiattino I, Silva C, Lolas F, Valdebenito C, Rodriguez E. Percepciones y estados emocionales sobre el proyecto genoma humano en actores sociales seleccionados en la Region Metropolitana, Chile. Revista Chilena de Salud Publica 2005; 9: 154-161.

31. Valdebenito C, Rodriguez M, Hidalgo A, Cardenas U, Lolas F. Salud intercultural: impacto en la identidad social de mujeres aimaras. Acta Bioethica 2006; 12(2): 185-191.

32. Valdebenito C. Definiendo Homo sapiens sapiens: aproximacion antropologica. Acta Bioethica 2007; 13(1): 71-78. 


\section{APPENDIX III}

\section{Academic events related to the program}

1. Seminar International: Ethical Guidelines for Research Involving Human Beings. New Developments, organized by CIEB and BU, with the sponsoring of Fogarty International Center, Santiago, Chile, October 2003.

2. International seminar: Fighting for the dignity of life: Ten years of bioethical debate, organized by CIEB, Heidelberg Center for Latin America and the Psychiatric Clinic of the University of Chile, Santiago, Chile, May 2004.

3. PAHO Knowledge Management and Learning Unit Video Conference Dialogue Health achievements - Extending social protection of health in America, co-organized by CIEB/BU, May 2004.

4. Video Conference: Integration of Ethics into Public Policies - The notion of ethical sustainability in health care and research, 10 years of bioethics at PAHO, organized by BU and Virtual University, Santiago, Chile, October 2004.

5. First Latin American Encounter of Oral Health and Bioethics, organized by the Faculty of Dentistry of the University of Chile, CIEB and BU, with the sponsoring of Fogarty International Center, Santiago, Chile, November 2004.

6. Video conference: Biotechnology, genomics and bioethics, their implications for public health, organized by CIEB, with the collaboration of the Hospital Clinic of the University of Chile, Center for Latin America of Heidelberg University at Chile, Center for Humanities of National Andres Bello University, World Bank Global Development Learning Network and Institute of Public Affairs of University of Chile, Santiago, Chile, July 2005.

7. Video conference: Health Priorities and Intercultural Health, organized by CIEB and the University of Toronto, with the collaboration of University Arturo Prat, Superintendence of Isapres, PAHO/WHO, Health Service Bio-Bio and Ministry of Health Chile, and University of McGill Canada, August 2006.

8. Bicentennial Program in Science and Technology Ethics and Innovation Technology, organized by CIEB University of Chile, with the collaboration of Pharmaceutic Industry Chamber and CONICYT, August 2006.

9. Second Latin American Encounter of Oral Health and Bioethics, organized by the Faculty of Dentistry of the University of Buenos Aires, Argentine Dental Association, CIEB and BP PAHO, with the sponsoring of Fogarty International Center, Buenos Aires, Argentine, September 2006.

10. Book presentation: Investigacion en Salud. Dimension Etica. University of Chile, September, 2006.

11. Bicentennial Program in Science and Technology Ethics and Innovation Technology. Towards a Social Dialogue in the Hidden Dimension of Knowledge Transfer, organized by CIEB University of Chile, with the collaboration of Pharmaceutic Industry Chamber and CONICYT, November, 2006.

12. CITI Program (Collaborative International Training Initiative) in Research Ethics, Ethics of Research in Internet, , Santiago, Chile, November 2006.

13. Book presentation: Etica de la Innovacion Tecnologica. University of Chile, January, 2007.

14. Ethics in Collaborative Research Program. Lima, Peru, May 2007.

15. Training in ethics of biomedical research and scientific ethical review committees for Latin American Regulatory Agencies staff. Santiago, Chile, June 2007.

16. Video Conference: Health Priorities and Intercultural Health, organized by CIEB and the University of Toronto with the collaboration of the Regional Program of Bioethics PAHO/WHO, Ministry of Health and CONICYT, July 2007.

17. Third Latin American Encounter of Oral Health and Bioethics, organized by the Faculty of Dentistry of the Cayetano Heredia University of Lima, CIEB and BP PAHO, Lima, Peru, December 2007.

18. International Forum Bioethical challenges in health: dignity, vulnerability and research. CIEB and 
Cristoforus Deneke - ISDEN Bioethics Association, University Garcilaso de la Vega, Lima, Peru, September 2008.

19. Book Presentation Health Priorities and Intercultural Health, Diego Portales University, July 2008.

20. Fourth Latin American Encounter of Oral Health and Bioethics, organized by CIEB and Circulo Argentino de Odontologia, Buenos Aires, November 2008.

21. International Conference: Health Regulation Ethics, Santiago, Chile, April 2009.

22. Seminar: Current Perspectives on Violence, Heidelberg University Latin American Center, Santiago, Chile, June 2009.

23. Presentation: Monographies Acta Bioethica. Heidelberg University Latin American Center, Santiago, Chile, November, 2009.

24. Seminar: Politics and Culture in Chile: A Prospective View, organized by CIEB and Center for Advanced Studies Peace and Truth (Madrasa), Catholic University, November 2010.

25. Presentation: Monography Acta Bioethica Anthropological Medicine and Nuremberg Trial. Heidelberg University Latin American Center, Santiago, Chile, January 2011.

26. Classification of cultural and scientific publications. Chilean Institute, Santiago, Chile, July and November 2011. 